\title{
Design, synthesis and biological evaluation of PSMA/hepsin-targeted heterobivalent ligands
}

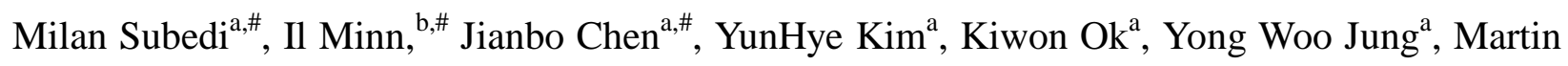
G. Pomper ${ }^{\mathrm{b}}$, Youngjoo Byun ${ }^{\mathrm{a}, \mathrm{b}, \mathrm{c}, *}$

${ }^{a}$ College of Pharmacy, Korea University, 2511 Sejong-ro, Jochiwon-eup, Sejong 339-700, South Korea

${ }^{\mathrm{b}}$ Department of Radiology, Johns Hopkins Medical Institution, 1550 Orleans street, Baltimore 21287, MD, USA

${ }^{\mathrm{c} B i o m e d i c a l}$ Research Center, Korea University Guro Hospital, 148 Gurodong-ro, Guro-gu, Seoul 152-703, South Korea

*Corresponding author:

Youngjoo Byun, Ph.D.

College of Pharmacy, Korea University, 2511 Sejong-ro, Jochiwon-eup, Sejong 339-700, South Korea.

Tel.: 82-44-860-1619; Fax: 82-44-860-1607; E-mail: yjbyun1@ korea.ac.kr

${ }^{\#}$ Equal contribution 


\begin{abstract}
Cell surface biomarkers such as prostate-specific membrane antigen (PSMA) and hepsin have received considerable attention as targets for imaging prostate cancer (PCa) due to their high cell surface expression in such tumors and easy access for imaging probes. Novel amidine-containing indole analogs (13-21) as hepsin inhibitors were designed and synthesized. These compounds showed in vitro inhibitory activity against hepsin with $\mathrm{IC}_{50}$ values from 5.9 to $70 \mu \mathrm{M}$. Based on the SAR of amidine-derived analogs, the novel heterobivalent compound 30, targeting both hepsin and PSMA, was synthesized by linking compound 18 with Lys-urea-Glu, the key scaffold for the specific binding to PSMA, followed by the conjugation of the optical dye sulfoCy7. Compound 30 exhibited inhibitory activities against PSMA and hepsin, with $\mathrm{IC}_{50}$ values of 28 $\mathrm{nM}$ and $2.8 \mu \mathrm{M}$, respectively. In vitro cell uptake and preliminary in vivo optical imaging studies of 30 showed selective binding and retention in both PSMA and hepsin high-expressing PC3/ML-PSMA-HPN cells as compared with low-expressing PC3/ML cells.
\end{abstract}

KEYWORDS: PSMA, Hepsin, Prostate Cancer, Heterobivalent Ligands, Molecular Imaging 


\section{Introduction}

Prostate cancer $(\mathrm{PCa})$ is the most common cancer in American men and the second leading cause of cancer death in this group [1]. The American Cancer Society estimates that there were approximately 220,800 new cases of PCa and 27,540 deaths from PCa in 2015 (www.cancer.org). The current gold standard for diagnosis of PCa is an elevated level of prostate-specific antigen (PSA) and abnormality of the prostate by digital rectal examination (DRE), followed by prostate needle biopsy [2]. However, benign conditions such as prostatitis and benign prostatic hyperplasia (BPH) can increase the level of PSA [3]. A comprehensive clinical study in Europe reported that PSA screening can lower the death risk from PCa, while a US study showed no statistical difference following PSA screening [4-6]. 76\% of patients with a raised PSA level are not diagnosed with $\mathrm{PCa}$, and $2 \%$ of patients with fast-growing $\mathrm{PCa}$ have a normal level of PSA (www.prostatecanceruk.org). Conversely, the effectiveness of DRE depends on the skill and experience of the examiner. $72-82 \%$ of individuals who undergo biopsy based on DRE findings are not diagnosed with PCa [7]. Therefore, there is an urgent need to explore novel biomarkers for the precise and efficient detection of PCa. Cell surface biomarkers including prostate-specific membrane antigen (PSMA), hepsin, and integrin- $\alpha_{v} \beta_{3}$ have received substantial attention as target proteins for the imaging of PCa due to their elevated cell surface expression on cancer cells compared with normal cells and easy access for imaging probes [8-14].

PSMA, a type II zinc-dependent protease, is highly expressed on the surface of prostate cancer cells as well as on the neovasculature of most solid tumors [15]. The active site of PSMA consists of two distinct sub-pockets, which form a 'glutamate-sensor' S1 site (pharmacophore) and an amphiphilic S1 site (non-pharmacophore). A cylindrical $\sim 20 \AA$ deep tunnel region exists adjacent to the $S 1$ site and projects towards the hydrophilic surface of the enzyme [16, 17]. The 
ability to detect overexpression of PSMA has provided novel avenues for the diagnosis and treatment of PCa. Hepsin is also a cell surface serine protease composed of 413 amino acids, with a 255-residue trypsin-like serine protease domain and a 109-residue region that forms a scavenger receptor cysteine-rich (SRCR) domain [18]. Hepsin is overexpressed in up to $90 \%$ of prostate tumors, with levels often increased $>10$ fold in metastatic PCa than in normal prostate or BPH [19-21]. PSMA and hepsin may represent excellent targets for PCa imaging since both have an enzymatic active site in the extracellular region. Imaging probes for the targets do not require penetration of the cell membrane, thus, have less chance of being degraded by metabolic enzymes.

Due to the heterogeneous and multifactorial nature of $\mathrm{PCa}$, the search for novel biomarkers is a key to precise diagnosis and efficient treatment [22]. Recent studies have shifted from the identification of individual biomarkers to the utilization of combinations of known specific markers [23]. A multi-targeting strategy has several advantages over single-targeting. Targeting multiple surface markers simultaneously can improve the sensitivity and specificity of detection through synergistic binding affinities to target proteins. The concept of targeting more than one cell surface protein for imaging purposes has been demonstrated by combining two biomarkers [22-24]. Dual-targeting was found to be more predictive with respect to distinguishing between $\mathrm{PCa}$ and $\mathrm{BPH}$ than when a single biomarker is targeted [22]. Eder and coworkers developed heterodimer molecules targeting both PSMA and the gastrin-releasing peptide receptor (GRPR), which exhibited high binding affinities to a cell line expressing both GRPR and PSMA [24]. More recently, Shallal and coworkers have developed heterobivalent agents targeting PSMA and integrin $\alpha_{\mathrm{v}} \beta_{3}[14]$.

We designed and synthesized a heterobivalent ligand which can bind to PSMA as well as 
hepsin. We used 2-(4-hydroxybiphenyl-3-yl)-1H-indole-5-carboximidamide as a hepsin-binding scaffold while Lys-urea-Glu moiety as a PSMA-binding scaffold. The amidine group was reported to interact with Asp189, a key amino acid in the hepsin active site [25]. Lys-urea-Glu moiety was reported to be strong binder to PSMA despite structural modification on the $\varepsilon$-amine of Lys. For in vitro cell uptake and in vivo imaging studies, we conjugated a PSMA-hepsin ligand with an optical dye SulfoCy7.

\section{Results and Discussion}

\subsection{Synthesis of hepsin-targeted analogs}

As shown in Scheme 1, hepsin-targeted amidine compounds (13-21) were synthesized in six steps using commercially available aniline, acetophenone, and arylboronic acids. Preparations of the intermediate imine 2 and the indole-5-carbonitrile $\mathbf{3}$ were achieved by applying the procedure previously reported by us [26]. Briefly, the $\mathrm{N}$-aryl imine $\mathbf{2}$ was prepared by reacting 4-aminobenzonitrile with 5'-bromo-2'-hydroxyacetophenone in toluene under piperidine-catalyzed basic condition. The indole-5-carbonitrile $\mathbf{3}$ was prepared from $\mathbf{2}$ using $\mathrm{Pd}(\mathrm{OAc})_{2}(20 \mathrm{~mol} \%)$ and $\mathrm{Bu}_{4} \mathrm{NBr}(2 \mathrm{eq})$ in $\mathrm{DMSO}$.

\section{< Scheme 1 >}

The amidine functional group had been reported to interact with the carboxylic acid present in the side chain of Asp 189 in hepsin. Although several methods for directly converting nitrile into an amidine group have been reported [27, 28], they were not suitable for the synthesis of a 
designed PSMA-hepsin conjugate which has labile groups under the reported conditions. Therefore, we utilized an oxadiazole ring as the amidine-protecting group, which can be removed under mild conditions $\left(\mathrm{H}_{2}\right.$, Raney-nickel). Reaction of $\mathbf{3}$ with hydroxylamine in ethanol afforded the oxime analog 4 in $60 \%$ yield. Compound 4 was subsequently cyclized to give the oxadiazole 5 using ethyl acetate and ethanol as solvents by modifying a reported procedure [29]. Reaction of $\mathbf{5}$ with appropriate arylboronic acids under Suzuki Coupling conditions at $100^{\circ} \mathrm{C}$ for 4 hours, with $\mathrm{Pd}\left(\mathrm{PPh}_{3}\right)_{4}$ as a catalyst, $\mathrm{Cs}_{2} \mathrm{CO}_{3}$ as a base, and $\mathrm{DMF} / \mathrm{H}_{2} \mathrm{O}$ (5:1) as a solvent, afforded the corresponding biphenyls 6-12. The oxadiazole-containing biphenyls were converted into the final amidines 13-21 by applying hydrogenation conditions $\left(\mathrm{H}_{2}\right.$, Raney-nickel, 50 psi) for 7 hours in a mixture of methanol and acetic acid (7:1) [30]. Deprotection of the oxadiazole ring proceeded in two steps. The $\mathrm{O}-\mathrm{N}$ bond of the oxadiazole ring is first cleaved, followed by the cleavage of $\mathrm{C}-\mathrm{N}$ bond. We observed partially-deprotected compounds during HPLC experiments, which have similar retention time to the fully-deprotected amidine compounds 13-21. However, the partially-deprotected compounds were hydrolyzed slowly at room temperature to give the corresponding amidines.

\subsection{Synthesis of the heterobivalent ligand}

The synthesis of the heterobivalent ligand $\mathbf{3 0}$ is outlined in Schemes 2 and 3. The use of protecting group for three hydrophilic functional groups (e.g., amidine, carboxylic acid and amine) was crucial for the preparation of $\mathbf{3 0}$. During the synthesis of $\mathbf{3 0}$, the oxadiazole ring as a protecting group of the amidine was introduced in the first step and removed at the final step because it was highly stable under acidic or basic reaction conditions and also be deprotected by hydrogenation without affecting the other functional groups. Deprotection of the Boc group of compound 9 was achieved using trifluoroacetic acid (TFA) to afford compound 22. Reaction of 
22 with Fmoc-Lys(Boc)-OH under traditional peptide-coupling conditions (HATU in DMF) afforded compound $\mathbf{2 3}$ in $56 \%$ yield. The Fmoc group of $\mathbf{2 3}$ was removed by treatment with $25 \%$ piperidine in DMF at room temperature to give compound 24. The free amine of $\mathbf{2 4}$ was conjugated with the tBu-protected Lys-urea-Glu 25, a known PSMA-binding scaffold [31], to provide compound $\mathbf{2 6}$ in $30 \%$ yield. Both the tBu and Boc groups of $\mathbf{2 6}$ were removed quantitatively using 50\% TFA in dichloromethane for 2 hours at room temperature to afford compound 27.

\section{< Scheme 2 >}

Transformation of the oxadiazole ring of $\mathbf{2 7}$ into the amidine group was achieved under hydrogenation conditions $\left(\mathrm{H}_{2}\right.$, Raney-nickel, 50 psi) for 4 hours in dioxane/water/acetic acid (6:2:1) by modifying a previously published procedure [30].

\section{< Scheme 3 >}

The hydrogenation reaction of the oxadiazole moiety generated two products, the amidine $\mathbf{2 8}$ and the partially-deprotected $\mathbf{2 9}$. The hydrogenation reaction time was critical for the deprotection of the oxadiazole ring. The optimal reaction time was found to be 4 hours, with reaction time longer than 4 hours generating more by-products through the cleavage of amide bonds. The commercially available SulfoCy7 NHS ester was conjugated to a mixture of $\mathbf{2 8}$ and $\mathbf{2 9}$ in Tris$\mathrm{HCl}$ buffer $(0.1 \mathrm{M}, \mathrm{pH} 8.5)$ at room temperature to afford the dye-linked PSMA-hepsin conjugates $\mathbf{3 0}$ and 31. Pure compounds $\mathbf{3 0}$ and $\mathbf{3 1}$ were obtained by reversed-phase HPLC, 
applying gradient conditions using $0.5 \%$ formic acid in acetonitrile (A) and water (B) as mobile phase (30\% to $40 \% \mathrm{~A}$ in $20 \mathrm{~min} ; 40 \%$ to $30 \% \mathrm{~A}$ in $25 \mathrm{~min}$ at $3.5 \mathrm{~mL} / \mathrm{min}$ flow rate) with a retention time of $5.79 \mathrm{~min}$ and $6.21 \mathrm{~min}$, respectively. Compound $\mathbf{3 1}$ was hydrolyzed slowly at room temperature to give compound $\mathbf{3 0}$.

\subsection{Molecular docking study}

The reported hepsin X-ray crystal structure (PDB ID: 1O5E) in complex with the amidinecontaining ligand (CA-14) was utilized as a template for the docking studies of the synthesized compounds 13-21 [25]. The ligand CA-14 presented strong hydrogen-bonding interactions with His57 and Ser195, which were known to be a part of the catalytic triad of hepsin. The positivelycharged amidine group made strong interactions with the carboxylic acid present in Asp189.

\section{$<$ Figure 1 >}

Docking studies of compounds 13-21 with hepsin revealed that they were located in the active site similar to the crystal ligand CA-14 [25]. As shown in Figure 1, the docked pose of compound 19 exhibited an additional interaction with Ser214 as compared with the original ligand.

\subsection{In vitro hepsin and PSMA inhibitory activities}

For in vitro hepsin assay, the known hepsin substrate Boc-Gln-Ala-Arg-AMC was used to determine the hepsin inhibitory activities of the synthesized compounds (13-21 and 30). The crystal ligand CA-14 (Figure 1) was synthesized by following a reported procedure [32] and used as a reference for in vitro hepsin assay. All of the synthesized compounds (13-21 and 30) showed 
inhibitory activity against hepsin similar to CA-14, with $\mathrm{IC}_{50}$ values from 5.9 to $70 \mu \mathrm{M}$ (Table 1). We observed that the amidine moiety is essential for hepsin-binding activity, since none of the compounds (5-12) with the oxadiazole ring showed hepsin activity. Even the bulky heterobivalent compound $\mathbf{3 0}$ exhibited similar hepsin-inhibitory activity $\left(\mathrm{IC}_{50}=2.8 \mu \mathrm{M}\right)$ to the monovalent compounds 13-21, indicating that the active site of hepsin tolerates the introduction of bulky groups.

\section{$<$ Table 1 >}

The PSMA inhibitory activities of the PSMA-hepsin conjugates $\mathbf{3 0}$ and $\mathbf{3 1}$ were determined by measuring the amount of glutamate released from the hydrolysis of $\mathrm{N}$-acetyl-Laspartyl-L-glutamate (NAAG). The $\mathrm{IC}_{50}$ values of $\mathbf{3 0}$ and $\mathbf{3 1}$ were $38 \mathrm{nM}$ and $25 \mathrm{nM}$,

respectively (Figure 2). Although they were less potent than the urea-based low molecular weight compound ZJ-43 presumably due to the addition of bulky fluorophore and hepsin binding moieties, $\mathbf{3 0}$ and $\mathbf{3 1}$ were still sufficiently potent for optical imaging. Previously, the bulky compounds, which consisted of the optical dyes and the PSMA-binding scaffold (Lys-urea-Glu) with $\mathrm{IC}_{50}$ values in the double-digit nanomolar concentration range, exhibited strong binding affinity for PSMA-expressing tumors in an in vivo study [33].

\section{$<$ Figure 2 >}

\subsection{In vitro cell uptake study}


Cell uptake studies revealed that $\mathbf{3 0}$ and $\mathbf{3 1}$ specifically bound to PC3/ML-PSMA (PSMA-expressing cell line), PC3/ML-HPN (hepsin-expressing cell line), and PC3/ML-PSMAHPN (PSMA- and hepsin-expressing cell line) cells (Figure 3). Both compounds exhibited strong binding affinity for PSMA-expressing cells even at a concentration of $10 \mathrm{nM}$ as compared with PC3/ML cells. The weak binding of the two compounds to PC3/ML-HPN cells might be due to their moderate affinity for hepsin. Both compounds also showed a slightly better uptake at higher concentrations by cells expressing both targets compared with cells expressing PSMA only, indicating synergistic affinity enhancement.

\section{$<$ Figure $3>$}

\subsection{Preliminary in vivo animal study}

We evaluated whether the dual-targeted heterobivalent compound $\mathbf{3 0}$ is capable of specifically targeting PSMA/hepsin-expressing tumors in vivo. We generated a subcutaneous xenograft model of PC3/ML-PSMA (red circle in Figure 4) and PC3/ML-PSMA-HPN (white circle in Figure 4), and injected the animal with $1 \mathrm{nmol}$ of 30. Near infrared (NIR) images were taken at $24 \mathrm{hr}$ post-injection, showing that $\mathbf{3 0}$ exhibited a higher uptake and retention in the PC3/ML-PSMA-HPN tumor than in the PC3/ML-PSMA tumor.

\section{$<$ Figure 4 >}

\section{Conclusion}

In summary, we designed, synthesized, and evaluated diverse amidine-functionalized analogs as hepsin inhibitors. In vitro studies showed that the amidine-containing compounds 1321 exhibited moderate affinity for hepsin. The PSMA-hepsin conjugate $\mathbf{3 0}$ was prepared by 
linking the identified hepsin-binding amidine scaffold with the PSMA-binding Lys-urea-Glu scaffold, followed by conjugation of the optical dye SulfoCy7. Compound $\mathbf{3 0}$ exhibited strong binding affinity for PSMA and moderate binding affinity for hepsin. In vitro cell uptake and preliminary in vivo animal optical imaging studies indicated that $\mathbf{3 0}$ have a potential to used as a lead compound in the development of more potent PSMA-hepsin conjugates which may provide a more powerful mechanism for imaging metastatic prostate cancer.

\section{Experimental Sections}

\subsection{Chemistry}

\subsubsection{General}

Chemicals and solvents used in the reaction were purchased from Aldrich, Acros, TCI, and Lumiprobe. ${ }^{1} \mathrm{H}$ NMR and ${ }^{13} \mathrm{C}$ NMR spectra were carried out on BRUKER Biospin AVANCE $300 \mathrm{MHz}$ and $75 \mathrm{MHz}$ spectrometer, respectively. Chemical shifts were reported as $\delta$ values downfield from internal TMS in appropriate organic solvents. Mass spectra were obtained on Agilent 6530 Accurate mass Q-TOF LC/MS spectrometer and Agilent 6460 Triple Quad LC/MS spectrometer. High performance liquid chromatography (HPLC) was carried out on Agilent 1290

Infinity LC using Gemini-NX C18 column (150 mm x 10 mm, $5 \mu \mathrm{m}, 110 \AA$ Å). Melting points were determined on microscope hot stage apparatus (Linkam THMS 6000). Hydrogenation was carried out using Parr-Hydrogenator (Marathon Electric). Silica gel column chromatography experiments were carried out using Merck Silica Gel F $F_{254}$.

\subsubsection{4-\{(E)-[1-(5-Bromo-2-hydroxyphenyl)ethylidene]amino\}benzonitrile (2)}

To a solution of 4-aminobenzonitrile $(1.00 \mathrm{~g}, 8.4 \mathrm{mmol})$ in toluene $(10 \mathrm{~mL})$ were added 5'- 
bromo-2'-hydroxyacetophenone $(1.80 \mathrm{~g}, 8.4 \mathrm{mmol})$ and piperidine $(1 \mathrm{~mL})$. The reaction mixture was stirred at $130{ }^{\circ} \mathrm{C}$ for $6 \mathrm{hr}$ using Dean-Stark apparatus. The reaction mixture was cooled to room temperature and excess solvent was concentrated to dryness under reduced pressure. The residue was washed with a mixture of hexane and ethylacetate $(25 \mathrm{~mL} \times 2)$ to afford compound 2 as yellow solid. Yield: 56\%, $\mathrm{R}_{\mathrm{f}}$ : 0.52 (hexane/EA = 9/1), mp: $145-148{ }^{\circ} \mathrm{C},{ }^{1} \mathrm{H}$ NMR $(300 \mathrm{MHz}$, $\left.\mathrm{CDCl}_{3}\right): \delta 13.70(\mathrm{~s}, 1 \mathrm{H}, \mathrm{NH}), 7.78-7.70(\mathrm{~m}, 3 \mathrm{H}, \mathrm{Ar}-\mathrm{H}), 7.50(\mathrm{dd}, 1 \mathrm{H}, \mathrm{Ar}-\mathrm{H}, J=8.7 \& 2.4 \mathrm{~Hz})$, $7.02(\mathrm{~d}, 2 \mathrm{H}, \mathrm{Ar}-\mathrm{H}, J=8.7 \mathrm{~Hz}), 6.95(\mathrm{~d}, 2 \mathrm{H}, \mathrm{Ar}-\mathrm{H}, J=9.0 \mathrm{~Hz}), 2.34$ (s, 3H, $\left.\underline{\mathrm{H}}_{3}\right)$, HRMS (ESI): [M-H] calcd for $\mathrm{C}_{15} \mathrm{H}_{11} \mathrm{BrN}_{2} \mathrm{O}: 313.0055$, found: 312.9987, ESI-MS/MS: 195.0436 [M-Br-CN$\mathrm{OH}-\mathrm{H}]^{-}$

\subsubsection{2-(5-Bromo-2-hydroxyphenyl)-1H-indole-5-carbonitrile (3)}

To a solution of compound $2(0.90 \mathrm{~g}, 2.9 \mathrm{mmol})$ in DMSO $(10 \mathrm{~mL})$ were added $\mathrm{Pd}(\mathrm{OAc})_{2}(65$ $\mathrm{mg}, 0.29 \mathrm{mmol})$ and $\mathrm{Bu}_{4} \mathrm{NBr}(1.87 \mathrm{~g}, 5.8 \mathrm{mmol})$. The mixture was stirred at $110{ }^{\circ} \mathrm{C}$ for $12 \mathrm{hr}$. Upon cooling to room temperature, the reaction mixture was diluted with water $(50 \mathrm{~mL})$ and ethyl acetate $(50 \mathrm{~mL})$. The combined organic phase was dried over $\mathrm{MgSO}_{4}$, filtered, and concentrated under reduced pressure. The crude residue was purified by silica gel chromatography to afford compound 3 as solid. Yield: $33 \%, \mathrm{R}_{\mathrm{f}}: 0.23$ (hexane/EA $=3 / 1$ ), $\mathrm{mp}$ : 103-105 ${ }^{\circ} \mathrm{C},{ }^{1} \mathrm{H}$ NMR (300 MHz, DMSO-d $\left.{ }^{6}\right): \delta 11.84$ (s, 1H, NH), 10.65 (s, 1H, OH), $8.07(\mathrm{~s}$, 1H, Ar-H), $7.95(\mathrm{~d}, 1 \mathrm{H}, \mathrm{Ar}-\mathrm{H}, J=8.7 \mathrm{~Hz}), 7.59(\mathrm{~d}, 1 \mathrm{H}, \mathrm{Ar}-\mathrm{H}, J=8.7 \mathrm{~Hz}), 7.45-7.32(\mathrm{~m}, 2 \mathrm{H}, \mathrm{Ar}-$ H), $7.22(\mathrm{~s}, 1 \mathrm{H}, \mathrm{Ar}-\mathrm{H}), 6.98(\mathrm{~d}, 1 \mathrm{H}, \operatorname{Ar}-\mathrm{H}, J=8.7 \mathrm{~Hz}), \mathrm{HRMS}(\mathrm{ESI}):[\mathrm{M}-\mathrm{H}]^{-}$calcd for $\mathrm{C}_{15} \mathrm{H}_{9} \mathrm{BrN}_{2} \mathrm{O}: 310.9898$, found: 310.9807, ESI-MS/MS: 231.0576 [M-Br-H]', 189.0467 [M-Br-

\section{$\mathrm{CN}-\mathrm{OH}-\mathrm{H}]^{-}$}




\subsection{4. (Z)-2-(5-Bromo-2-hydroxyphenyl)-N'-hydroxy-1H-indole-5-carboximidamide (4)}

To a solution of compound $3(0.37 \mathrm{~g}, 1.2 \mathrm{mmol})$ in ethanol $(20 \mathrm{~mL})$ were added hydroxylamine hydrochloride $(0.71 \mathrm{~g}, 10.2 \mathrm{mmol})$ and sodium carbonate $(0.55 \mathrm{~g}, 5.1 \mathrm{mmol})$. The reaction mixture was stirred at $90{ }^{\circ} \mathrm{C}$ for $7 \mathrm{hr}$. After the completion of reaction, excess solvent was evaporated to dryness under reduced pressure. The residue was diluted with water $(25 \mathrm{~mL})$ and ethyl acetate $(25 \mathrm{~mL})$. The combined organic phase was dried over $\mathrm{MgSO}_{4}$, filtered, and concentrated under reduced pressure. The crude residue was purified by silica gel chromatography to afford compound $\mathbf{4}$ as solid. Yield: $60 \%, \mathrm{R}_{\mathrm{f}}: 0.12($ hexane/EA $=3 / 1)$, mp: > $400{ }^{\circ} \mathrm{C}, \quad{ }^{1} \mathrm{H}$ NMR $\left(300 \mathrm{MHz}\right.$, DMSO-d $\left.\mathrm{d}^{6}\right): \delta 11.37$ (s, 1H, OH), 9.39 (s, $\left.1 \mathrm{H}, \mathrm{NH}\right), 8.22(\mathrm{~s}, 1 \mathrm{H}$, Ar-H), $7.93(\mathrm{~d}, 1 \mathrm{H}, \mathrm{Ar}-\mathrm{H}, J=2.4 \mathrm{~Hz}), 7.83(\mathrm{~d}, 1 \mathrm{H}, \mathrm{Ar}-\mathrm{H}, J=1.2 \mathrm{~Hz}), 7.49-7.38(\mathrm{~m}, 2 \mathrm{H}, \mathrm{Ar}-\mathrm{H})$, $7.28(\mathrm{dd}, 1 \mathrm{H}, \mathrm{Ar}-\mathrm{H}, J=2.4 \& 8.4 \mathrm{~Hz}), 7.10(\mathrm{~s}, 1 \mathrm{H}, \mathrm{Ar}-\mathrm{H}), 6.94(\mathrm{~d}, 1 \mathrm{H}, \mathrm{Ar}-\mathrm{H}, J=8.7 \mathrm{~Hz}), 5.71(\mathrm{~s}$, $2 \mathrm{H}, \mathrm{N}_{2}$ ), HRMS (ESI): [M-H] calcd for $\mathrm{C}_{15} \mathrm{H}_{12} \mathrm{BrN}_{3} \mathrm{O}_{2}$ : 344.0113, found: 344.0068, ESI-

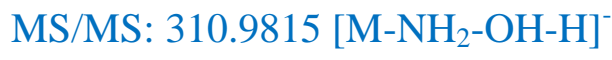

\subsubsection{4-Bromo-2-[5-(5-methyl-1,2,4-oxadiazol-3-yl)-1H-indol-2-yl]phenol (5)}

To a solution of compound $4(0.23 \mathrm{~g}, 0.69 \mathrm{mmol})$ in ethanol $(9 \mathrm{~mL})$ were added sodium ethoxide (90 mg, $1.32 \mathrm{mmol})$ and ethyl acetate $(1 \mathrm{~mL})$. The reaction mixture was stirred at $90{ }^{\circ} \mathrm{C}$ for $2 \mathrm{hr}$. After completion of reaction, excess solvent was evaporated to dryness under reduced pressure. The residue was diluted with water $(25 \mathrm{~mL})$ and ethyl acetate $(25 \mathrm{~mL})$. The organic phase was dried over $\mathrm{MgSO}_{4}$, filtered, and concentrated under reduced pressure. The crude residue was purified by silica gel chromatography to afford compound $\mathbf{5}$ as solid. Yield: $42 \%, \mathrm{R}_{\mathrm{f}}$ : 0.25 (hexane/EA = 3/1), mp: $236-238{ }^{\circ} \mathrm{C},{ }^{1} \mathrm{H}$ NMR $\left(300 \mathrm{MHz}, \mathrm{DMSO}-\mathrm{d}^{6}\right): \delta 11.61(\mathrm{~s}, 1 \mathrm{H}, \mathrm{NH}), 10.59$ (s, 1H, OH), $8.22(\mathrm{~s}, 1 \mathrm{H}, \mathrm{Ar}-\mathrm{H}), 7.95(\mathrm{~d}, 1 \mathrm{H}, \mathrm{Ar}-\mathrm{H}, J=2.4 \mathrm{~Hz}), 7.74(\mathrm{dd}, 1 \mathrm{H}, \mathrm{Ar}-\mathrm{H}, J=8.4$ \& 
$1.5 \mathrm{~Hz}), 7.58(\mathrm{~d}, 1 \mathrm{H}, \mathrm{Ar}-\mathrm{H}, J=8.4 \mathrm{~Hz}), 7.33(\mathrm{dd}, 1 \mathrm{H}, \mathrm{Ar}-\mathrm{H}, J=2.4 \& 8.7 \mathrm{~Hz}), 7.21(\mathrm{~s}, 1 \mathrm{H}, \mathrm{Ar}-$ H), $\left.6.97(\mathrm{~d}, 1 \mathrm{H}, \mathrm{Ar}-\mathrm{H}, J=8.7 \mathrm{~Hz}), 2.66\left(\mathrm{~s}, 3 \mathrm{H}, \mathrm{C}_{3}\right),\right),{ }^{13} \mathrm{C}$ NMR $\left(75 \mathrm{MHz}\right.$, DMSO- $\left.d_{6}\right): \delta$ $117.17,154.31,138.36,135.51,131.38,129.78,128.68,121.10,120.72,120.28,119.00,117.82$ 112.54, 111.16, 103.52, $12.49\left(\underline{\mathrm{CH}}_{3}\right)$, HRMS (ESI): [M-H] ${ }^{-}$calcd for $\mathrm{C}_{17} \mathrm{H}_{12} \mathrm{BrN}_{3} \mathrm{O}_{2}:$ 368.0113, found: 368.0025, ESI-MS/MS: 327.1152 [M-Ac-OH-H] ${ }^{-}$

\subsubsection{4'-Chloro-3-[5-(5-methyl-1,2,4-oxadiazol-3-yl)-1H-indol-2-yl][1,1'-biphenyl]-4-ol (6)}

To a solution of compound 5 (100 mg, $0.27 \mathrm{mmol})$ in DMF $(5 \mathrm{~mL})$ were added 4-chlorophenyl boronic acid (42 mg, $0.27 \mathrm{mmol})$, cesium carbonate (182 $\mathrm{mg}, 0.55 \mathrm{mmol}$ in $1 \mathrm{~mL}$ water) and $\operatorname{Pd}\left(\mathrm{PPh}_{3}\right)_{4}(16 \mathrm{mg}, 0.014 \mathrm{mmol})$. The reaction mixture was stirred at $100^{\circ} \mathrm{C}$ for $4 \mathrm{hr}$. Upon cooling to room temperature, the reaction mixture was diluted with water $(30 \mathrm{~mL})$ and ethyl acetate $(15 \mathrm{~mL})$. The solution $\mathrm{pH}$ was adjusted to 7 by using $1 \mathrm{~N} \mathrm{HCl}$. The organic phase was dried over $\mathrm{MgSO}_{4}$, filtered, and concentrated under reduced pressure. The residue was purified by silica gel chromatography to afford compound $\mathbf{6}$. Yield: $30 \%, \mathrm{R}_{\mathrm{f}}: 0.25$ (hexane/EA = 3/1), LRMS (ESI): [M-H] ${ }^{-}$calcd for $\mathrm{C}_{23} \mathrm{H}_{16} \mathrm{ClN}_{3} \mathrm{O}_{2}: 400.1$, found: 400.1, ESI-MS/MS: 290.1 [M-Ph$\mathrm{Cl}-\mathrm{H}]^{-}$

\subsubsection{2-[5-(5-Methyl-1,2,4-oxadiazol-3-yl)-1H-indol-2-yl]-4-(thiophen-2-yl)phenol (7)}

In the same method as 6, 2-thienylboronic acid was used instead of 4-chlorophenylboronic acid. Yield: $33 \%, \mathrm{R}_{\mathrm{f}}: 0.25$ (hexane/EA = 3/1), LRMS (ESI): [M-H] calcd for $\mathrm{C}_{21} \mathrm{H}_{15} \mathrm{~N}_{3} \mathrm{O}_{2} \mathrm{~S}: 372.1$, found: 372.0, ESI-MS/MS: $315.0\left[\mathrm{M}-\mathrm{Ac}-\mathrm{NH}_{2}\right]^{-}$

4.1.8. 4'-Fluoro-3-[5-(5-methyl-1,2,4-oxadiazol-3-yl)-1H-indol-2-yl] ][1,1'-biphenyl]-4-ol (8) 
In the same method as 6, 4-fluorophenyboronic acid was used instead of 4-chlorophenylboronic acid. Yield: $34 \%, \mathrm{R}_{\mathrm{f}}: 0.27$ (hexane/EA= 3/1), LRMS (ESI): $[\mathrm{M}-\mathrm{H}]^{-}$calcd for $\mathrm{C}_{23} \mathrm{H}_{16} \mathrm{FN}_{3} \mathrm{O}_{2}$ : 384.1, found: 384.1, ESI-MS/MS: 290.1 [M-Ph-F-H]

\subsection{9. tert-Butyl(\{4'-hydroxy-3'-[5-(5-methyl-1,2,4-oxadiazol-3-yl)-1H-indol-2-yl][1,1'-} biphenyl]-4-yl\}methyl)carbamate (9)

To a solution of 4-aminomethylphenylboronic acid $(2.65 \mathrm{mmol})$ in THF $(10 \mathrm{~mL})$ were added ditert-butyl dicarbonate $(3.97 \mathrm{mmol})$ and triethylamine $(1 \mathrm{~mL})$. The mixture was stirred at room temperature for $1 \mathrm{hr}$. After completion of the reaction, excess solvent was evaporated to dryness under reduced pressure. The residue was diluted with water $(30 \mathrm{~mL})$ and ethyl acetate $(15 \mathrm{~mL})$. The organic phase was separated, dried over $\mathrm{MgSO}_{4}$, filtered, and concentrated under reduced pressure. The NHBoc-protected boronic acid was then used for Suzuki coupling as described in compound 6 to afford compound 9 . Yield: $32 \%, \mathrm{R}_{\mathrm{f}}$ : 0.31 (hexane/EA= 3/2), $\mathrm{mp}: 147-148{ }^{\circ} \mathrm{C},{ }^{1} \mathrm{H}$ NMR (300 MHz, DMSO-d ${ }^{6}$ ): $\delta 11.63$ (br s, 1H, NH), 10.39 (br s, 1H, OH), 8.22 (s, 1H, Ar-H), 8. 07 (d, 1H, Ar-H, $J=2.4$ Hz), 7.76-7.64 (m, 3H, Ar-H), 7.59 (d, 1H, Ar-H, J = 8.4 Hz), 7.51-7.42 (m, 2H, Ar-H), $7.32(\mathrm{~d}, 2 \mathrm{H}, \mathrm{Ar}-\mathrm{H}, J=8.1 \mathrm{~Hz}), 7.23(\mathrm{~s}, 1 \mathrm{H}, \mathrm{Ar}-\mathrm{H}), 7.10$ (d, 1H, Ar-H, J=8.4 Hz), $4.17\left(\mathrm{~d}, 2 \mathrm{H}, J=6.0 \mathrm{~Hz}, \mathrm{C}_{2}\right), 2.66\left(\mathrm{~s}, 3 \mathrm{H}, \mathrm{OCH}_{3}\right), 1.41$ (s, 9H, $\left.\left(\mathrm{C}_{3}\right)_{3}\right), \operatorname{HRMS}(\mathrm{ESI}):[\mathrm{M}-\mathrm{H}]^{-}$ calcd for $\mathrm{C}_{29} \mathrm{H}_{28} \mathrm{~N}_{4} \mathrm{O}_{4}$ : 495.2111, found: 495.1981, ESI-MS/MS: 439.1448 [M-Ac- $\left.\mathrm{NH}_{2}\right]^{-}$, $395.1550[\mathrm{M}-\mathrm{Boc}-\mathrm{H}]^{-}$

\subsubsection{3-[5-(5-Methyl-1,2,4-oxadiazol-3-yl)-1H-indol-2-yl][1,1'-biphenyl]-4-ol (10)}

In the same method as $\mathbf{6}$, phenyboronic acid was used instead of 4-chlorophenylboronic acid. Yield: $37 \%, \mathrm{R}_{\mathrm{f}}: 0.23$ (hexane/EA= 3/1), LRMS (ESI): $[\mathrm{M}-\mathrm{H}]^{-}$calcd for $\mathrm{C}_{23} \mathrm{H}_{17} \mathrm{~N}_{3} \mathrm{O}_{2}: 366.1$, 
found:366.1

\subsubsection{4'-Methoxy-3-[5-(5-methyl-1,2,4-oxadiazol-3-yl)-1H-indol-2-yl][1,1'-biphenyl]-4-ol (11)}

In the same method as 6, 4-methoxyphenyboronic acid was used instead of 4chlorophenylboronic acid. Yield: 36\%, $\mathrm{R}_{\mathrm{f}}: 0.27$ (hexane/EA= 3/1), LRMS (ESI): [M-H] ${ }^{-}$calcd for $\mathrm{C}_{24} \mathrm{H}_{19} \mathrm{~N}_{3} \mathrm{O}_{3}: 396.1$, found: 396.2

\subsubsection{3-[5-(5-Methyl-1,2,4-oxadiazol-3-yl)-1H-indol-2-yl]-4'-(trifluoromethyl)[1,1'-biphenyl]-} 4-ol (12)

In the same method as 6, 4-trifluoromethyphenyboronic acid was used instead of 4chlorophenylboronic acid. Yield: 39\%, $\mathrm{R}_{\mathrm{f}}: 0.26$ (hexane/EA=3/1), LRMS (ESI): $[\mathrm{M}-\mathrm{H}]^{-}$calcd for $\mathrm{C}_{24} \mathrm{H}_{16} \mathrm{~F}_{3} \mathrm{~N}_{3} \mathrm{O}_{2}: 434.1$, found: 434.1

\subsubsection{2-(4'-Cloro-4-hydroxy [1,1'-biphenyl]-3-yl)-1H-indole-5-carboximidamide (13)}

To a solution of compound 5 in a mixture of $\mathrm{MeOH}$ and $\mathrm{AcOH}(10 \mathrm{~mL}, 7: 1)$ was added a catalytic amount of Raney-nickel. The reaction mixture was shaken under 50 psi of $\mathrm{H}_{2}$ for $7 \mathrm{hr}$. The mixture was filtered through Celite and the filtrate was concentrated to dryness under reduced pressure. The residue was purified by reversed-phase HPLC to afford compound $\mathbf{1 3}$. HPLC: $80 / 20$ water/acetonitrile ( $0.1 \%$ formic acid), flow rate $2 \mathrm{~mL} / \mathrm{min}, \mathrm{R}_{\mathrm{t}}=9.15 \mathrm{~min}$, Yield: 22\%, mp: $126-128^{\circ} \mathrm{C},{ }^{1} \mathrm{H}$ NMR (300 MHz, $\left.\mathrm{CD}_{3} \mathrm{OD}\right): \delta 8.58$ (s, $\left.2 \mathrm{H}, \mathrm{N}_{2}\right), 8.11(\mathrm{~s}, 1 \mathrm{H}, \mathrm{Ar}-\mathrm{H})$, $8.01(\mathrm{~d}, 1 \mathrm{H}, \mathrm{Ar}-\mathrm{H}, J=2.4 \mathrm{~Hz}), 7.67-7.57(\mathrm{~m}, 3 \mathrm{H}, \mathrm{Ar}-\mathrm{H}), 7.54-7.45(\mathrm{~m}, 1 \mathrm{H}, \mathrm{Ar}-\mathrm{H}), 7.45-7.25(\mathrm{~m}$, $4 \mathrm{H}, \mathrm{Ar}-\mathrm{H}), 6.99$ (d, 1H, Ar-H, $J=8.4 \mathrm{~Hz}),{ }^{13} \mathrm{C}$ NMR $\left(75 \mathrm{MHz}, \mathrm{CD}_{3} \mathrm{OD}\right): 168.92,168.03,158.67$, $140.43,139.78,139.22,131.78,129.40,128.60,128.37,127.37,127.30,125.45,120.19,119.33$ 
118.53, 117.93, 111.33. HRMS (ESI): $[\mathrm{M}+\mathrm{H}]^{+}$calcd for $\mathrm{C}_{21} \mathrm{H}_{16} \mathrm{ClN}_{3} \mathrm{O}$ : 362.0982, found: 362.1053, ESI-MS/MS: $345.0763\left[\mathrm{M}-\mathrm{NH}_{3}+\mathrm{H}\right]^{+}, 320.0793\left[\mathrm{M}-\mathrm{NH}_{3}-\mathrm{CN}+\mathrm{H}\right]^{+}$

\subsubsection{2-[2-Hydroxy-5-(thiophen-2-yl)phenyl]-1H-indole-5-carboximidamide (14)}

In the same method as $\mathbf{1 3}$, compound $\mathbf{1 4}$ was prepared from $\mathbf{7}$. HPLC: 70/30 water/acetonitrile (0.1\% formic acid), flow rate $2 \mathrm{~mL} / \mathrm{min} ; \mathrm{R}_{\mathrm{t}}=8.71 \mathrm{~min}$, Yield: $37 \%$, mp: $240-243{ }^{\circ} \mathrm{C},{ }^{1} \mathrm{H} \mathrm{NMR}$ (300 MHz, CD $\left.{ }_{3} \mathrm{OD}\right): \delta 8.58$ (s, 4H), $8.14(\mathrm{~s}, 1 \mathrm{H}, \mathrm{Ar}-\mathrm{H}), 8.04$ (d, 1H, Ar-H, $\left.J=2.1 \mathrm{~Hz}\right), 7.66(\mathrm{~d}$, $1 \mathrm{H}, \mathrm{Ar}-\mathrm{H}, J=8.4 \mathrm{~Hz}), 7.56-7.50(\mathrm{~m}, 1 \mathrm{H}, \mathrm{Ar}-\mathrm{H}), 7.47(\mathrm{dd}, 1 \mathrm{H}, \mathrm{Ar}-\mathrm{H}, J=8.4 \& 2.4 \mathrm{~Hz}), 7.37-$ $7.28(\mathrm{~m}, 2 \mathrm{H}, \mathrm{Ar}-\mathrm{H}), 7.19$ (s, 1H, Ar-H), 7.13-7.04 (m, 1H, Ar-H), $7.00(\mathrm{~d}, 1 \mathrm{H}, \mathrm{Ar}-\mathrm{H}, J=8.4 \mathrm{~Hz})$

${ }^{13} \mathrm{C}$ NMR (75 MHz, $\left.\mathrm{CD}_{3} \mathrm{OD}\right): 168.99,167.98,154.86,144.01,139.61,138.61,128.45,127.59$, $126.45,124.78,123.34,121.80,120.54,119.79,118.51,118.22,116.90,111.54$, HRMS (ESI): $[\mathrm{M}+\mathrm{H}]^{+}$calcd for $\mathrm{C}_{19} \mathrm{H}_{15} \mathrm{~N}_{3} \mathrm{OS}$ : 334.0936, found: 334.1013, ESI-MS/MS: 317.0727 [M$\left.\mathrm{NH}_{3}+\mathrm{H}\right]^{+}, 292.0788\left[\mathrm{M}-\mathrm{NH}_{3}-\mathrm{CN}+\mathrm{H}\right]^{+}$

\subsubsection{2-(4-Hydroxy[1,1'-biphenyl]-3-yl)-1H-indole-5-carboximidamide (15)}

In the same method as $\mathbf{1 3}$, compound $\mathbf{1 5}$ was prepared from 5. HPLC: acetonitrile(A)/water(B) ( $0.1 \%$ formic acid), the gradient consisted of $10 \%$ A to $90 \%$ A over $30 \mathrm{~min}$ at $2 \mathrm{~mL} / \mathrm{min}$ flow rate. $\mathrm{R}_{\mathrm{t}}=9.78$ min. Yield: $46 \%$, mp: $181-183{ }^{\circ} \mathrm{C},{ }^{1} \mathrm{H}$ NMR $\left(300 \mathrm{MHz}, \mathrm{CD}_{3} \mathrm{CN} / \mathrm{D}_{2} \mathrm{O}\right): \delta 8.48(\mathrm{~s}, 1 \mathrm{H}$,

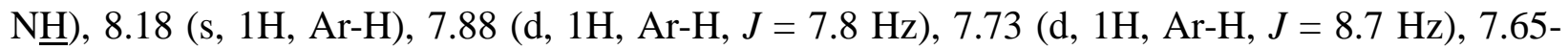
7.56 (m, 1H, Ar-H), 7.39-7.29 (m, 1H, Ar-H), 7.12 (s, 1H, Ar-H), 7.15-6.95 (m, 2H, Ar-H), ${ }^{13} \mathrm{C}$ NMR (75 MHz, $\left.\mathrm{CD}_{3} \mathrm{CN} / \mathrm{D}_{2} \mathrm{O}\right): 170.52,167.61,154.28,139.82,139.29,130.23,128.61,121.63$, 121.43, 121.04, 120.88, 118.22, 117.18, 112.62, 101.09, HRMS (ESI): $[\mathrm{M}+\mathrm{H}]^{+}$calcd for $\mathrm{C}_{15} \mathrm{H}_{13} \mathrm{~N}_{3} \mathrm{O}: 252.1059$, found: 252.1134, ESI-MS/MS: $235.0863\left[\mathrm{M}-\mathrm{NH}_{3}+\mathrm{H}\right]^{+}, 210.0902[\mathrm{M}-$ 
$\left.\mathrm{NH}_{3}-\mathrm{CN}+\mathrm{H}\right]^{+}$

\subsubsection{2-(4'-Fluoro-4-hydroxy[1,1'-biphenyl]-3-yl)-1H-indole-5-carboximidamide (16)}

In the same method as $\mathbf{1 3}$, compound $\mathbf{1 6}$ was prepared from $\mathbf{8}$. HPLC: acetonitrile(A)/water(B) ( $0.1 \%$ formic acid), the gradient consisted of $40 \%$ A to $50 \%$ A over 30 min at $2 \mathrm{~mL} / \mathrm{min}$ flow rate. $\mathrm{R}_{\mathrm{t}}=9.78$ min. Yield: $30 \%$, mp: $165-168{ }^{\circ} \mathrm{C},{ }^{1} \mathrm{H}$ NMR (300 MHz, $\left.\mathrm{CD}_{3} \mathrm{OD}\right): \delta 8.14$ (s, $\left.1 \mathrm{H}, \mathrm{Ar}-\mathrm{H}\right)$, $8.03(\mathrm{~d}, 1 \mathrm{H}, \mathrm{Ar}-\mathrm{H}, J=2.1 \mathrm{~Hz}), 7.68-7.60(\mathrm{~m}, 3 \mathrm{H}, \mathrm{Ar}-\mathrm{H}), 7.57-7.45(\mathrm{~m}, 2 \mathrm{H}, \mathrm{Ar}-\mathrm{H}), 7.37(\mathrm{~d}, 1 \mathrm{H}$, Ar-H, $J=8.1 \mathrm{~Hz}), 7.22(\mathrm{~s}, 1 \mathrm{H}, \mathrm{Ar}-\mathrm{H}), 7.06(\mathrm{~d}, 1 \mathrm{H}, \mathrm{Ar}-\mathrm{H}, J=8.4 \mathrm{~Hz}),{ }^{13} \mathrm{C}$ NMR $(75 \mathrm{MHz}$, $\left.\mathrm{CD}_{3} \mathrm{OD}\right): 167.95,163.79,154.07,139.67,138.65,137.00,132.10,128.56,128.49,128.00$, $127.90,127.33,125.88,120.60,119.83,118.40,118.19,116.55,115.17,114.89,111.53,101.26$, HRMS (ESI) MS: $[\mathrm{M}+\mathrm{H}]^{+}$calcd for $\mathrm{C}_{21} \mathrm{H}_{16} \mathrm{FN}_{3} \mathrm{O}$ : 346.1277, found: 346.1361, ESI-MS/MS: $330.2389\left[\mathrm{M}-\mathrm{NH}_{3}+\mathrm{H}\right]^{+}, 305.2931\left[\mathrm{M}-\mathrm{NH}_{3}-\mathrm{CN}+\mathrm{H}\right]^{+}$

\subsubsection{7. tert-Butyl\{[3'-(5-carbamimidoyl-1H-indol-2-yl)-4'-hydroxy[1,1'-biphenyl]-4-yl] methyl\}} carbamate (17)

In the same method as $\mathbf{1 3}$, compound $\mathbf{1 7}$ was prepared from $\mathbf{9}$. HPLC: 70/30 water/acetonitrile (0.1\% formic acid), flow rate $2 \mathrm{~mL} / \mathrm{min}, \mathrm{R}_{\mathrm{t}}=5.94 \mathrm{~min}$, Yield: $40 \%, \mathrm{mp}: 196-199{ }^{\circ} \mathrm{C},{ }^{1} \mathrm{H}$ NMR (300 MHz, CD 3 OD): $\delta 8.14$ (s, 1H, Ar-H), $8.00(\mathrm{~d}, 1 \mathrm{H}, \mathrm{Ar}-\mathrm{H}, J=2.1 \mathrm{~Hz}), 7.71-7.63(\mathrm{~m}, 3 \mathrm{H}, \mathrm{Ar}-$ H), 7.57-7.52 (m, 1H, Ar-H), $7.45(\mathrm{dd}, 1 \mathrm{H}, \mathrm{Ar}-\mathrm{H}, J=8.4 \& 2.1 \mathrm{~Hz}), 7.24-7.14(\mathrm{~m}, 3 \mathrm{H}, \mathrm{Ar}-\mathrm{H})$, $7.07(\mathrm{~d}, 1 \mathrm{H}, \mathrm{Ar}-\mathrm{H}, J=8.4 \mathrm{~Hz}), 4.23\left(\mathrm{~s}, 2 \mathrm{H}, \mathrm{C}_{2}\right), 1.49\left(\mathrm{~s}, 9 \mathrm{H},\left(\mathrm{CH}_{3}\right)_{3}\right),{ }^{13} \mathrm{C}$ NMR $(75 \mathrm{MHz}$, $\left.\mathrm{CD}_{3} \mathrm{OD}\right): 167.97,157.22,154.03,139.68,139.33,138.77,138.12,132.80,128.59,128.51$, $127.34,127.25,126.19,125.83,120.58,119.79,118.34,118.16,116.51,111.52,101.20,78.82$, $43.35\left(\underline{\mathrm{C}} \mathrm{H}_{2}\right), 27.38\left(\underline{\mathrm{CH}_{3}}\right)_{3}$, HRMS (ESI) MS: $[\mathrm{M}+\mathrm{H}]^{+}$calcd for $\mathrm{C}_{27} \mathrm{H}_{28} \mathrm{~N}_{4} \mathrm{O}_{3}: 457.2162$, found: 457.2225, ESI-MS/MS: $357.3035[\mathrm{M}-\mathrm{Boc}+\mathrm{H}]^{+} 341.2742\left[\mathrm{M}-\mathrm{Boc}-\mathrm{NH}_{2}+\mathrm{H}\right]^{+}, 324.2468$ [M-Boc- 
$\left.\mathrm{NH}_{2}-\mathrm{NH}_{3}+\mathrm{H}\right]^{+}, 299.2524$ [M-Boc- $\left.\mathrm{NH}_{2}-\mathrm{NH}_{3}-\mathrm{CN}+\mathrm{H}\right]^{+}$

\subsubsection{2-[4'-(Aminomethyl)-4-hydroxy[1,1'-biphenyl]-3-yl]-1H-indole-5-carboximidamide (18)}

Compound 17 (10 mg, $0.02 \mathrm{mmol})$ was dissolved in a mixture of TFA and dichloromethane (2 $\mathrm{mL}, 1: 1)$. The reaction mixture was stirred at room temperature for $1 \mathrm{hr}$. The mixture was concentrated to dryness under reduced pressure. The residue was dissolved in a mixture of water and acetonitrile and purified by reversed-phase HPLC to give compound 18. HPLC: 70/30 water/acetonitrile ( $0.1 \%$ formic acid), flow rate $2 \mathrm{~mL} / \mathrm{min}, \mathrm{R}_{\mathrm{t}}=2.56 \mathrm{~min}$. Yield: $72 \%$, mp: $126-$ $129{ }^{\circ} \mathrm{C},{ }^{1} \mathrm{H}$ NMR $\left(300 \mathrm{MHz}, \mathrm{CD}_{3} \mathrm{CN} / \mathrm{D}_{2} \mathrm{O}\right): \delta 8.49(\mathrm{~d}, 2 \mathrm{H}, \mathrm{Ar}-\mathrm{H}, J=7.2 \mathrm{~Hz}), 8.14$ (d, 1H, Ar-H, $J=8.1 \mathrm{~Hz}), 8.06(\mathrm{~d}, 1 \mathrm{H}, \mathrm{Ar}-\mathrm{H}, J=8.7 \mathrm{~Hz}), 8.01-7.87(\mathrm{~m}, 4 \mathrm{H}, \mathrm{Ar}-\mathrm{H}), 7.61(\mathrm{~s}, 1 \mathrm{H}, \mathrm{Ar}-\mathrm{H}), 7.53$ (d, $1 \mathrm{H}, \mathrm{Ar}-\mathrm{H}, J=8.4 \mathrm{~Hz}), 4.54\left(\mathrm{~s}, 2 \mathrm{H}, \mathrm{C}_{2}\right),{ }^{13} \mathrm{C} \mathrm{NMR}\left(75 \mathrm{MHz}, \mathrm{CD}_{3} \mathrm{CN} / \mathrm{D}_{2} \mathrm{O}\right): 167.57,161.90$, $154.34,141.20,139.86,132.61,131.91,130.12,128.62,127.50,126.89,121.53,121.05,117.79$, 112.66, 101.63, $43.15\left(\underline{\mathrm{CH}}_{2}\right)$, HRMS (ESI) MS: $[\mathrm{M}+\mathrm{H}]^{+}$calcd for $\mathrm{C}_{22} \mathrm{H}_{20} \mathrm{~N}_{4} \mathrm{O}: 357.1637$, found: 357.1711, ESI-MS/MS: $340.2968\left[\mathrm{M}-\mathrm{NH}_{3}+\mathrm{H}\right]^{-}, 323.2406\left[\mathrm{M}-\mathrm{NH}_{3}-\mathrm{NH}_{3}+\mathrm{H}\right]^{+}, 315.2702[\mathrm{M}-$ $\left.\mathrm{NH}_{3}-\mathrm{CN}+\mathrm{H}\right]^{+}$

\subsubsection{2-(4-Hydroxy[1,1'-biphenyl]-3-yl)-1H-indole-5-carboximidamide (19)}

In the same method as 13, compound 19 was prepared from 10. HPLC: 72/28 water/acetonitrile (0.1\% formic acid), flow rate $2 \mathrm{~mL} / \mathrm{min}, \mathrm{R}_{\mathrm{t}}=8.62 \mathrm{~min}$, Yield: $42 \%, \mathrm{mp}: 220-223{ }^{\circ} \mathrm{C},{ }^{1} \mathrm{H}$ NMR (300 MHz, $\left.\mathrm{CD}_{3} \mathrm{CN} / \mathrm{D}_{2} \mathrm{O}\right): \delta 8.76(\mathrm{~s}, 1 \mathrm{H}, \mathrm{N} \underline{\mathrm{H}}), 8.48(\mathrm{~s}, 1 \mathrm{H}, \mathrm{Ar}-\mathrm{H}), 8.43$ (s, 1H, Ar-H), 8.10-7.97 (m, 3H, Ar-H), 7.96-7.80 (m, 5H, Ar-H), 7.78-7.70 (m, 1H, Ar-H), 7.57 (s, 1H, Ar-H), 7.49 (d,

$1 \mathrm{H}, \mathrm{Ar}-\mathrm{H}, J=8.4 \mathrm{~Hz}),{ }^{13} \mathrm{C}$ NMR $\left(75 \mathrm{MHz}, \mathrm{CD}_{3} \mathrm{CN} / \mathrm{D}_{2} \mathrm{O}\right): 153.94,140.49,139.78,138.84$, 134.31, 133.52, 131.24, 129.45, 128.29, 127.57, 127.36, 126.91, 126.77, 121.41, 120.94, 117.67, 
112.98, HRMS (ESI) MS: $[\mathrm{M}+\mathrm{H}]^{+}$calcd for $\mathrm{C}_{21} \mathrm{H}_{17} \mathrm{~N}_{3} \mathrm{O}$ : 328.1372, found: 328.1435, ESIMS/MS: $311.2381\left[\mathrm{M}-\mathrm{NH}_{3}+\mathrm{H}\right]^{+}, 286.2379\left[\mathrm{M}-\mathrm{NH}_{3}-\mathrm{CN}+\mathrm{H}\right]^{+}$

\subsubsection{2-(4-Hydroxy-4'-methoxy[1,1'-biphenyl]-3-yl)-1H-indole-5-carboximidamide (20)}

In the same method as 13, compound $\mathbf{2 0}$ was prepared from 11. HPLC: 75/25 water/acetonitrile( $0.1 \%$ formic acid), flow rate $2 \mathrm{~mL} / \mathrm{min}, \mathrm{R}_{\mathrm{t}}=12.98 \mathrm{~min}$, Yield: $33 \%$, mp: 132 -

$135{ }^{\circ} \mathrm{C},{ }^{1} \mathrm{H}$ NMR $\left(300 \mathrm{MHz}, \mathrm{CD}_{3} \mathrm{CN} / \mathrm{D}_{2} \mathrm{O}\right): \delta 8.48(\mathrm{~s}, 1 \mathrm{H}, \mathrm{N} \underline{\mathrm{H}}), 8.19(\mathrm{~s}, 1 \mathrm{H}, \mathrm{Ar}-\mathrm{H}), 8.08(\mathrm{~d}, 1 \mathrm{H}$, Ar-H, $J=2.4 \mathrm{~Hz}), 7.80-7.68(\mathrm{~m}, 3 \mathrm{H}, \mathrm{Ar}-\mathrm{H}), 7.65-7.52(\mathrm{~m}, 2 \mathrm{H}, \mathrm{Ar}-\mathrm{H}), 7.29(\mathrm{~s}, 1 \mathrm{H}, \mathrm{Ar}-\mathrm{H}), 7.22-$

$7.10(\mathrm{~m}, 3 \mathrm{H}, \mathrm{Ar}-\mathrm{H}), 6.92-6.82(\mathrm{~m}, 1 \mathrm{H}, \mathrm{Ar}-\mathrm{H}), 3.85\left(\mathrm{~s}, 3 \mathrm{H}, \mathrm{OC}^{\mathrm{H}}\right),{ }^{13} \mathrm{C}$ NMR $(75 \mathrm{MHz}$, $\left.\mathrm{CD}_{3} \mathrm{CN} / \mathrm{D}_{2} \mathrm{O}\right): 172.68,169.92,161.54,156.06,142.12,135.61,135.43,130.91,130.83,130.41$, $128.58,123.75,123.23,120.0,118.67,117.63,117.11,114.88,103.69,57.94\left(\mathrm{OCH}_{3}\right)$, HRMS (ESI): $[\mathrm{M}+\mathrm{H}]^{+}$calcd for $\mathrm{C}_{22} \mathrm{H}_{19} \mathrm{~N}_{3} \mathrm{O}_{2}: 358.1477$, found: 358.1564 , ESI-MS/MS: 341.2541 [M$\left.\mathrm{NH}_{3}+\mathrm{H}\right]^{+}, 326.2285\left[\mathrm{M}-\mathrm{NH}_{3}-\mathrm{OH}+\mathrm{H}\right]^{+}, 316.2550\left[\mathrm{M}-\mathrm{NH}_{3}-\mathrm{CN}+\mathrm{H}\right]^{+}, 298.2271\left[\mathrm{M}-\mathrm{NH}_{3}-\mathrm{CN}-\right.$ $\mathrm{OH}+\mathrm{H}]^{+}$

4.1.21. 2-[4-Hydroxy-4'-(trifluoromethyl)[1,1'-biphenyl]-3-yl]-1H-indole-5-carboximidamide (21)

In the same method as 13, compound 21 was prepared from 12. HPLC: 68/32 water/acetonitrile( $0.1 \%$ formic acid), flow rate $2 \mathrm{~mL} / \mathrm{min}, \mathrm{Rt}=9.92 \mathrm{~min}$. Yield: $28 \%$, mp: 191 $193{ }^{\circ} \mathrm{C},{ }^{1} \mathrm{H}$ NMR $\left(300 \mathrm{MHz}, \mathrm{CD}_{3} \mathrm{CN} / \mathrm{D}_{2} \mathrm{O}\right): \delta 8.53$ (s, 2H, Ar-H), 8.29 (d, 2H, Ar-H, J = 7.5 Hz), $8.19(\mathrm{~d}, 2 \mathrm{H}, \mathrm{Ar}-\mathrm{H}, J=7.8 \mathrm{~Hz}), 8.12-7.90(\mathrm{~m}, 4 \mathrm{H}, \mathrm{Ar}-\mathrm{H}), 7.63(\mathrm{~s}, 1 \mathrm{H}, \mathrm{Ar}-\mathrm{H}), 7.56$ (d, 1H, Ar-H, $J$ $=8.4 \mathrm{~Hz}),{ }^{13} \mathrm{C} \mathrm{NMR}\left(75 \mathrm{MHz}, \mathrm{CD}_{3} \mathrm{CN} / \mathrm{D}_{2} \mathrm{O}\right): 167.59,154.84,144.48,139.88,131.86,128.79$, $127.48,127.22,126.28,121.56,121.10,117.88,112.68,101.78$, LRMS (ESI): $[\mathrm{M}+\mathrm{H}]^{+}$calcd for 
$\mathrm{C}_{22} \mathrm{H}_{16} \mathrm{~F}_{3} \mathrm{~N}_{3} \mathrm{O}: 396.1245$, found: 396.1272, ESI-MS/MS: $379.2376\left[\mathrm{M}-\mathrm{NH}_{3}+\mathrm{H}\right]^{+}, 354.2366[\mathrm{M}-$ $\left.\mathrm{NH}_{3}-\mathrm{CN}+\mathrm{H}\right]^{+}$

4.1.22. 4'-(Aminomethyl)-3-[5-(5-methyl-1,2,4-oxadiazol-3-yl)-1H-indol-2-yl][1,1'-biphenyl]-4ol (22)

Compound 9 (300 mg, $0.60 \mathrm{mmol}$ ) was dissolved in a mixture of TFA and dichloromethane (5 $\mathrm{mL}, 1: 1)$. The reaction mixture was stirred at room temperature for $1 \mathrm{hr}$. The mixture was concentrated under reduced pressure and used for next step without further purification. LRMS (ESI): [M-H] ${ }^{-}$calcd for $\mathrm{C}_{24} \mathrm{H}_{20} \mathrm{~N}_{4} \mathrm{O}_{2}: 395.1$, found: 395.0

\subsubsection{3. (9H-Fluoren-9-yl)methyl $N$-\{1-[(\{4'-hydroxy-3'-[5-(5-methyl-1,2,4-oxadiazol-3-yl)-1H-} indol-2-yl]-[1,1'-biphenyl]-4-yl\}methyl)carbamoyl]-5-\{[(propan-2-yloxy)carbonyl]amino\} pentyl\}carbamate (23)

To a solution of compound $22(100 \mathrm{mg}, 0.26 \mathrm{mmol})$ in DMF $(15 \mathrm{~mL})$ were added HATU (192 $\mathrm{mg}, 0.50 \mathrm{mmol})$, Fmoc-Lys(Boc)-OH (140 mg, $0.3 \mathrm{mmol})$, and triethylamine (70 $\mu \mathrm{l}, 0.50 \mathrm{mmol})$. The reaction mixture was stirred at room temperature for $8 \mathrm{hr}$. The reaction mixture was diluted with water $(30 \mathrm{~mL})$ and ethyl acetate $(15 \mathrm{~mL})$. The solution $\mathrm{pH}$ was adjusted to 7 by using $1 \mathrm{~N}$ $\mathrm{HCl}$. The organic phase was dried over $\mathrm{MgSO}_{4}$, filtered and concentrated under reduced pressure. The crude residue was purified by silica gel chromatography to afford compound $\mathbf{2 3}$. Yield: 56\%, $\mathrm{R}_{\mathrm{f}}: 0.41$ (hexane/EA= 3/2), ${ }^{1} \mathrm{H}$ NMR $\left(300 \mathrm{MHz}, \mathrm{DMSO}-\mathrm{d}^{6}\right): \delta 11.84(\mathrm{~s}, 1 \mathrm{H}, \mathrm{NH}), 8.52-8.45(\mathrm{~m}$, 1H, Ar-H), 8.26 (s, 1H, Ar-H), 8.09 (d, 1H, Ar-H, J = 12 Hz), 7.96 (s, 4H, Ar-H), 7.87 (d, 3H, Ar$\mathrm{H}, J=7.5 \mathrm{~Hz}), 7.81-7.62$ (m, 6H, Ar-H), 7.54 (d, 2H, Ar-H, $J=8.4 \mathrm{~Hz}), 7.43-7.20$ (m, 9H, Ar-H), $7.06(\mathrm{~s}, 1 \mathrm{H}, \mathrm{Ar}-\mathrm{H}), 6.82-6.74(\mathrm{~m}, 1 \mathrm{H}, \mathrm{Ar}-\mathrm{H}), 4.46-4.32(\mathrm{~m}, 4 \mathrm{H}), 4.30-4.20(\mathrm{~m}, 4 \mathrm{H}), 4.08-3.95(\mathrm{~m}$, 2H), $2.69(\mathrm{~s}, 1 \mathrm{H}), 2.59\left(\mathrm{~s}, 3 \mathrm{H}, \mathrm{C}_{3}\right), 1.35\left(\mathrm{~s}, 9 \mathrm{H},\left(\mathrm{C}_{3}\right)_{3}\right)$ 
4.1.24. tert-Butyl N-\{5-amino-5-[(\{4'-hydroxy-3'-[5-(5-methyl-1,2,4-oxadiazol-3-yl)-1H-indol-2yl][1,1'-biphenyl]-4-yl]tmethyl)carbamoyl]pentyl\}carbamate (24)

Compound 23 (50 mg, $0.06 \mathrm{mmol}$ ) was dissolved in 25\% piperidine in DMF (5 mL) and stirred at room temperature for $1 \mathrm{hr}$. The mixture was concentrated to dryness under reduced pressure and used for next step without purification. LRMS (ESI): $[\mathrm{M}+\mathrm{Na}]^{+}$calcd for $\mathrm{C}_{35} \mathrm{H}_{40} \mathrm{~N}_{6} \mathrm{O}_{5}: 647.3$, found: 647.4

\subsubsection{1,5-Di-tert-butyl 2-(\{[1-(tert-butoxy)-6-\{7-[(5-\{[(tert-butoxy)carbonyl]amino\}-1-[(\{4'-} hydroxy-3'-[5-(5-methyl-1,2,4-oxadiazol-3-yl)-1H-indol-2-yl][1,1'-biphenyl]-4-yl]methyl) carbamoyl]pentyl)carbamoyl]heptanamido\}-1-oxohexan-2-yl]carbamoyl\}amino)pentanedioate (26)

To a solution of compound $24(50 \mathrm{mg}, 0.08 \mathrm{mmol})$ in DMF $(10 \mathrm{~mL})$ were added trimethylamine $(0.05 \mathrm{~mL})$ and compound $25(59 \mathrm{mg}, 0.08 \mathrm{mmol})$ which was prepared by applying a reported procedure [31]. The mixture was stirred at room temperature for $1 \mathrm{hr}$. The reaction mixture was diluted with water $(30 \mathrm{~mL})$ and ethyl acetate $(15 \mathrm{~mL})$. The solution $\mathrm{pH}$ was adjusted to 7 by using $1 \mathrm{~N} \mathrm{HCl}$. The combined organic phase was dried over $\mathrm{MgSO}_{4}$, filtered and concentrated under reduced pressure. The crude residue was purified by silica gel chromatography to afford compound 26. Yield: $30 \%, \mathrm{R}_{\mathrm{f}}: 0.38$ (ethylacetate/methanol= 10/1), ${ }^{1} \mathrm{H}$ NMR (300 MHz, $\left.\mathrm{CD}_{3} \mathrm{OD}\right): \delta 8.28(\mathrm{~s}, 1 \mathrm{H}), 8.05-7.92(\mathrm{~m}, 1 \mathrm{H}, \mathrm{Ar}-\mathrm{H})$, 7.88-7.54 (m, 1H, Ar-H), 7.52-7.60 (m, 3H, Ar-H), 7.59-7.50 (m, 2H, Ar-H), 7.48-7.32 (m, 3H, Ar-H), 7.17-7.10 (m, 2H, Ar-H), 6.90-6.70 (m, $1 \mathrm{H}), 6.52-6.64(\mathrm{~m}, 1 \mathrm{H}), 6.42-6.30(\mathrm{~m}, 2 \mathrm{H}), 4.51-4.41(\mathrm{~m}, 3 \mathrm{H}), 4.39-4.32(\mathrm{~m}, 2 \mathrm{H}), 4.29(\mathrm{~s}, 1 \mathrm{H})$, 4.23-4.09 (m, 7H), 3.22-3.10 (m, 6H), 3.08-2.98 (m, 5H), 2.71-2.62 (m, 4H), 2.45-2.23 (m, 11H), 2.20-2.10 (m, 8H), 2.08-1.97 (m, 4H), 1.58-1.70 (m, 18H), 1.56-1.10 (m, 36H) 
4.1.26. 2-[(\{5-[7-(\{5-Amino-1-[(\{4'-hydroxy-3'-[5-(5-methyl-1,2,4-oxadiazol-3-yl)-1H-indol-2yl][1,1'-biphenyl]-4-yl]methyl)carbamoyl]pentyl\}carbamoyl)heptanamido]-1-carboxypentyl\} carbamoyl)amino]pentanedioic acid (27)

Compound 26 (20 mg, $0.02 \mathrm{mmol})$ was dissolved in a mixture of TFA and dichloromethane (5 $\mathrm{mL}, 1: 1)$ and stirred at room temperature for $2 \mathrm{hr}$. The mixture was concentrated under reduced pressure. The residue was purified by reversed-phase HPLC to afford compound 27. HPLC: $72 / 28$ water/acetonitrile ( $0.1 \%$ formic acid), flow rate $2 \mathrm{~mL} / \mathrm{min} ; \mathrm{Rt}=11.68 \mathrm{~min}$. Yield: $25 \%, \mathrm{mp}$ : 205-207 ${ }^{\circ} \mathrm{C},{ }^{1} \mathrm{H}$ NMR $\left(300 \mathrm{MHz}, \mathrm{CD}_{3} \mathrm{CN} / \mathrm{D}_{2} \mathrm{O}\right): \delta 8.16(\mathrm{~s}, 1 \mathrm{H}, \mathrm{Ar}-\mathrm{H}), 7.94(\mathrm{~s}, 1 \mathrm{H}, \mathrm{Ar}-\mathrm{H}), 7.60(\mathrm{~d}$, $1 \mathrm{H}, \mathrm{Ar}-\mathrm{H}, J=8.7 \mathrm{~Hz}), 7.53$ (t, 3H, Ar-H, $J=7.5 \mathrm{~Hz}), 7.40(\mathrm{~d}, 1 \mathrm{H}, \mathrm{Ar}-\mathrm{H}, J=8.4 \mathrm{~Hz}), 7.25(\mathrm{~d}, 2 \mathrm{H}$, Ar-H, $J=9.0 \mathrm{~Hz}), 7.05(\mathrm{~m}, 2 \mathrm{H}, \mathrm{Ar}-\mathrm{H}), 4.29(\mathrm{~s}, 2 \mathrm{H}), 3.98(\mathrm{~m}, 2 \mathrm{H}), 2.96(\mathrm{t}, 2 \mathrm{H}, J=6.8 \mathrm{~Hz}), 2.81$ (t, $2 \mathrm{H}, J=7.7 \mathrm{~Hz}), 2.53(\mathrm{~s}, 3 \mathrm{H}), 2.29(\mathrm{t}, 2 \mathrm{H}, J=7.5 \mathrm{~Hz}), 2.15(\mathrm{t}, 2 \mathrm{H}, J=7.4 \mathrm{~Hz}), 1.98(\mathrm{t}, 2 \mathrm{H}, J$ $=7.2 \mathrm{~Hz}), 1.58-1.82(\mathrm{~m}, 24 \mathrm{H}),{ }^{13} \mathrm{C} \mathrm{NMR}\left(75 \mathrm{MHz}, \mathrm{CD}_{3} \mathrm{CN} / \mathrm{D}_{2} \mathrm{O}\right): 180.44,178.72,178.33,176.1$ $7,171.72,156.20,141.78,140.69,140.03,139.85,135.31,131.01,130.59,130.35,129.34,128.7$ $9,123.13,120.17,119.94,114.83,56.44,56.21,56.05,45.07,41.88,41.57,38.45,38.21,34.19$, $33.42,33.18,30.98,30.86,30.80,30.19,28.98,28.04,27.98,25.25,25.07,14.37$, HRMS (ESI): $[\mathrm{M}+\mathrm{H}]^{+}$calcd for $\mathrm{C}_{50} \mathrm{H}_{63} \mathrm{~N}_{9} \mathrm{O}_{12}: 982.4596$, found: 982.4715, ESI-MS/MS: 835.4162 [M-Boc$\left.\mathrm{CH}_{3}(\mathrm{C}=\mathrm{O}) \mathrm{N}+\mathrm{H}\right]^{+}, 809.4351\left[\mathrm{M}-\mathrm{Boc}-\mathrm{CH}_{3}(\mathrm{C}=\mathrm{O}) \mathrm{N}-\mathrm{CN}+\mathrm{H}\right]^{+}$

4.1.27. 2-(\{[5-(7-\{[5-Amino-1-(\{[3'-(5-carbamimidoyl-1H-indol-2-yl)-4'-hydroxy-[1,1'biphenyl]-4-yl]methyl\}carbamoyl)pentyl]carbamoyl\}heptanamido)-1-carboxypentyl]carbamoyl\} amino)pentanedioic acid (28) and (Z)-4-(4-aminobutyl)-1-(4'-hydroxy-3'-(5-(N-(1hydroxyethylidene)carbamimidoyl)-1H-indol-2-yl)-[1,1'-biphenyl]-4-yl)-3,6,13,21-tetraoxo2,5,14,20,22-pentaazapentacosane-19,23,25-tricarboxylic acid (29)

To a solution of compound $\mathbf{2 7}$ in a mixture of dioxane, water, and acetic acid (5 mL, 6:2:1) was added catalytic amount of Raney-nickel. The mixture was shaken under $50 \mathrm{psi}$ of $\mathrm{H}_{2}$ for $4 \mathrm{hr}$. The 
mixture was filtered through Celite and the filtrate was concentrated to dryness under reduced pressure. The crude residue was purified by reversed-phase HPLC to afford a mixture of compounds 28 and 29. HPLC: acetonitrile (A)/water (B) (0.1\% formic acid). The gradient consisted of $10 \%$ A to $35 \%$ A over $8 \mathrm{~min} ; 35 \%$ A to $10 \%$ A over $10 \mathrm{~min}$ at $2 \mathrm{~mL} / \mathrm{min}$ flow rate, $\mathrm{Rt}=8.36$ min, Yield: $30 \%$, HRMS (ESI) compound 28: $[\mathrm{M}-\mathrm{H}]^{-}$calcd for $\mathrm{C}_{48} \mathrm{H}_{63} \mathrm{~N}_{9} \mathrm{O}_{11}: 940.4647$, found: 940.4680, HRMS (ESI) compound 29: [M-H] $]^{-}$calcd for $\mathrm{C}_{50} \mathrm{H}_{65} \mathrm{~N}_{9} \mathrm{O}_{12}$ : 982.4753, found: 982.4783

\subsubsection{1-(5-\{[5-(\{[3'-(5-Carbamimidoyl-1H-indol-2-yl)-4'-hydroxy-[1,1'-biphenyl]-4-yl]} methylcarbamoyl)-5-\{7-[(5-carboxy-5-\{[(1,3-dicarboxypropyl)carbamoyl]amino\}entyl) carbamoyl]heptanamido\}pentyl]carbamoyl $\}$ pentyl)-3,3-dimethyl-2-[(E)-2-[(3E)-3-\{2-[(2E)1,3,3-trimethyl-5-sulfonato-2,3-dihydro-1H-indol-2-ylidene] ethylidene fcyclohex-1-en-1yl]ethenyl]-3H-indol-1-ium-5-sulfonate (30) and 1-\{5-[(5-\{7-[(5-carboxy-5-\{[(1,3dicarboxypropyl)carbamoyl]amino\}pentyl)carbamoyl] heptanamido\}-5-(\{[4'-hydroxy-3'-(5-\{[(Z)(1-hydroxyethylidene)amino]methanimidoyl\}-1H-indol-2-yl)-[1,1'-biphenyl]-4-yl]methyl\} carbamoyl)pentyl)carbamoyl]pentyl\}-3,3-dimethyl-2-[(E)-2-[(3E)-3-\{2-[(2E)-1,3,3-trimethyl-5sulfonato-2,3-dihydro-1H-indol-2-ylidene ] ethylidene f cyclohex-1-en-1-yl]ethenyl]-3H-indol-1ium-5-sulfonate (31)

To a mixture of compounds $\mathbf{2 8}$ and $\mathbf{2 9}$ (2 $\mathrm{mg}$ in $0.3 \mathrm{~mL}$ of DMSO) were added tris- $\mathrm{HCl}$ buffer (0.3 mL, 0.1 M, pH=8.5) and Sulfo-Cyanine 7 NHS ester (2 $\mathrm{mg}$ in $0.1 \mathrm{~mL}$ of DMSO). The reaction mixture was stirred at room temperature for $4 \mathrm{hr}$. The residue was purified by reversed-phase HPLC to afford compounds $\mathbf{3 0}$ and 31, respectively. HPLC: mobile phase acetonitrile (A)/water (B) ( $0.1 \%$ formic acid). The gradient consisted of $30 \%$ to $40 \%$ A over 20 $\min ; 40 \%$ to $30 \%$ A over $25 \mathrm{~min}$ at $3.5 \mathrm{~mL} / \mathrm{min}$ flow rate, $\mathrm{Rt}=5.18 \mathrm{~min}$ for $\mathbf{3 0}, 6.21 \mathrm{~min}$ for 31 . Yield: $37 \%$ (30), 45\% (31), compound 30: LRMS (ESI) $[\mathrm{M}+\mathrm{H}]^{+}$calcd for $\mathrm{C}_{85} \mathrm{H}_{104} \mathrm{~N}_{11} \mathrm{O}_{18} \mathrm{~S}_{2}$ : 1631.7 found: $[\mathrm{M}+3 \mathrm{H}]^{3+} 544.8,[\mathrm{M}+2 \mathrm{H}]^{2+} 816.8$, and $[\mathrm{M}+\mathrm{H}]^{+}$1631.7; HRMS (ESI): $[\mathrm{M}+3 \mathrm{H}]^{3+}$ 
545.3593, $[\mathrm{M}+3 \mathrm{Na}]^{3+}$ 567.3402, ESI-MS/MS: 370.3259 [M-(Lys-urea-Glu)-2SO3-NH3$\mathrm{CN}+3 \mathrm{H}]^{3+}$, compound 31: LRMS (ESI) $[\mathrm{M}+\mathrm{H}]^{+}$calcd for $\mathrm{C}_{87} \mathrm{H}_{106} \mathrm{~N}_{11} \mathrm{O}_{19} \mathrm{~S}_{2}: 1674.9$, found: $[\mathrm{M}+3$ $\mathrm{H}]^{3+} 558.8,[\mathrm{M}+2 \mathrm{H}]^{2+} 838.0$ and $[\mathrm{M}+\mathrm{H}]^{+} 1674.7$

\subsection{Biological evaluation}

\subsubsection{In vitro hepsin-inhibitory assay}

The synthesized compounds $(10 \mathrm{nM}-10 \mu \mathrm{M})$ were diluted in $2 \%$ DMSO and mixed with activated hepsin (\#4776-SE-010, R\&D Systems, Minneapolis, Minnesota) to a 96-well plate (REF 353219; BD Falcon). The final assay concentration for hepsin was $0.3 \mathrm{nM}$ in TNC buffer (25 mM Tris, $150 \mathrm{mM} \mathrm{NaCl}, 5 \mathrm{mM} \mathrm{CaCl}, 2,01 \%$ Triton X-100, $\mathrm{pH}$ 8). After incubation for 30 min at room temperature Boc-QAR-AMC substrate (\#ES014, R\&D Systems, Minneapolis, Minnesota) was added to the Hepsin assays. The final substrate concentration was $150 \mu \mathrm{M}$ in final reaction volume of $100 \mu \mathrm{L}$. Changes in fluorescence (excitation at $380 \mathrm{~nm}$ and emission at $460 \mathrm{~nm}$ ) were measured at room temperature over time in a Biotek Synergy 2 plate reader (Molecular devices). From a plot of the mean reaction velocity versus comppound concentration, a non-linear four parameter curve fit was performed using GraphPad Prism version 4.03 for Windows (GraphPad Software, SanDiego, CA, www.graphpad.com) to determine inhibitor $\mathrm{IC}_{50}$.

\subsubsection{In vitro PSMA-inhibitory assay}

The $\mathrm{IC}_{50}$ values of compounds $\mathbf{3 0}$ and $\mathbf{3 1}$ were measured using NAALADase assay where the glutamic acid/glutamate released from the natural substrate of PSMA was analyzed by the fluorescence-based Amplex Red Glutamic Acid/Glutamate Oxidase assay kit (Invitrogen). In vitro PSMA binding affinities of the synthesized compounds were evaluated by following the 
manufacturer's procedure (www.lifetechnologies.com). Briefly, in a 96-well half area black flat bottom polystyrene NBS microplate (Corning, Tewksbury, MA), PSMA (3 nM, $10 \mu \mathrm{L}$ ) and different concentrations of inhibitor $(20 \mu \mathrm{L})$ or buffer (TBS, $20 \mu \mathrm{L})$ were incubated at $37^{\circ} \mathrm{C}$ for $30 \mathrm{~min}$. Then NAAG $(4 \mu \mathrm{M}, 10 \mu \mathrm{L})$ was added to the mixture $\left(37^{\circ} \mathrm{C}, 30 \mathrm{~min}\right)$. The fluorescence signals generated from the Amplex working solution $(50 \mu \mathrm{L})$ with the NAAG-liberated glutamate $\left(37^{\circ} \mathrm{C}, 1 \mathrm{~h}\right)$ were then quantified by the Synergy $\mathrm{H} 4$ hybrid multi-mode microplate reader (excitation at $530 \mathrm{~nm}$ and emission at $590 \mathrm{~nm}$ ). Data was analyzed by non-linear regression with GraphPad Prism 4.03 for Windows to determine inhibitor $\mathrm{IC}_{50}$.

\subsubsection{In vitro cell uptake study}

PC3/ML, PC3/ML-HPN, PC3/ML-PSMA and PC3/ML-PSMA-HPN cells were prepared at Johns Hopkins Medical Institutions, Baltimore, Maryland. The cells were seeded at a density of $5 \times 10^{5}$ cells/well in 6-well plates for $48 \mathrm{~h}$ until total adhesion was achieved. Then the medium was removed, and $\mathbf{3 0}$ and $\mathbf{3 1}$ (final concentration: $10 \mu \mathrm{g} / \mathrm{mL}$ ) in RPMI 1640 medium supplemented with 10\% FBS were added. After another $4 \mathrm{~h}$ of incubation, the cells were washed with cold PBS twice and fixed with $4 \%$ paraformaldehyde for 10 min and the flow cytometry (LSRII, BD Biosciences, San Jose, CA) analysis was employed to estimate the cellular uptake at different cell lines. Data were processed by FlowJo software (Ashland, OR)

\subsubsection{In vivo near infrared (NIR) optical imaging study}

One million cells of PC3/ML-PSMA and PC3/ML-PSMA-HPN were subcutaneously injected into upper flanks of six-week old male NSG (NOD/Shi-scid/IL-2R $\gamma^{\text {null }}$ ) mice. Two weeks after the cell injection, one nanomole of $\mathbf{3 0}$ in $100 \mathrm{uL}$ of PBS was injected via tail vein. 
Images were taken $24 \mathrm{hr}$ post injection of $\mathbf{3 0}$ using the Pearl Impulse Small Animal Imaging System (LI-COR, Lincoln, NA).

\subsection{In silico molecular docking studies}

\subsubsection{Ligand preparation and optimization}

All ligands 13-21 were generated as 2D and 3D structure by ChemBioDraw (ver. 11.0.1) and Chem3D Pro (ver. 11.0.1), respectively. The prepared compounds and CA-14, the original crystal ligand of hepsin X-ray crystal structure (PDB ID: 1O5E), were saved as .sdf file. The process of ligand preparation and optimization was performed by 'Sanitize' preparation protocol in SYBYL-X 2.1.1 (Tripos Inc., St Louis) to clean up the structures involving filling valences, standardizing, removing duplicates and producing only one molecule per input structure.

\subsubsection{Protein preparation}

The hepsin structure of in PDB format was downloaded from RCSB protein data bank (PDB ID: 1O5E). Structure preparation tool in SYBYL-X 2.1.1 was employed for the protein preparation. The original crystal ligand and water molecules were removed from the proteinligand complexes for docking. Conflicted side chains of amino acid residues were fixed. Hydrogen atoms were added under the application of TRIPOS Force Field as a default setting. Minimization process was performed by POWELL method, initial optimization option was changed to None from a default setting SIMPLEX, and termination gradient and max iteration were set $0.5 \mathrm{kcal} /\left(\mathrm{mol}^{*} \AA\right)$ and 1000 times, respectively.

\subsubsection{Molecular docking}

Docking studies of all prepared ligands were performed by Surflex-Dock Geom module in 
SYBYL-X 2.1.1. Docking was guided by the Surflex-Dock protomol, an idealized representation of a ligand that makes every potential interaction with the binding site. The binding site of hepsin X-ray crystal structure (PDB ID: 1O5E) for Surflex-Dock was defined according to the location information of CA-14, the original ligand. Two factors related with a generation of Protomol are

Bloat $(\AA)$ and Threshold were set to 0.5 and 0 , respectively. The maximum number of generated poses per each ligand and the minimum RMSD between final poses were set to 20 and 0.05 , respectively. Other parameters were applied with its default settings in all runs. The Surflex-Dock scoring function, which contains hydrophobic, polar, repulsive, entropic and solvation terms, was trained to estimate the dissociation constant $\left(\mathrm{K}_{\mathrm{d}}\right)$ expressed in $-\log \left(\mathrm{K}_{\mathrm{d}}\right)$ units. The docked poses were obtained after running Surflex-Dock and the scores of the docked poses were ranked according to total score and Cscore (Table 2: Supplementary data).

\section{Acknowledgments}

This research supported by the NIH (CA134675 and CA184228 to M.G.P), USA Department of Defense (W81XWH-10-1-0189 to Y.B), the National Research Foundation of Korea (NRF) (2014R1A1A2056522 and 2014R1A4A1007304 to Y. B). 


\section{References}

[1] R. Siegel, J. Ma, Z. Zou, A. Jemal, Cancer statistics, 2014, CA Cancer J. Clin., 64 (2014) 9-29.

[2] A. Horwich, C. Parker, C. Bangma, V. Kataja, E.G.W. Group, Prostate cancer: ESMO Clinical Practice Guidelines for diagnosis, treatment and follow-up, Ann. Oncol., 21 Suppl 5 (2010) v129-133.

[3] M.E. Ford, S.L. Havstad, R. Demers, C. Cole Johnson, Effects of false-positive prostate cancer screening results on subsequent prostate cancer screening behavior, Cancer Epidemiol. Biomarkers Prev. 14 (2005) 190-194.

[4] E.A.M. Heijnsdijk, E.M. Wever, A. Auvinen, J. Hugosson, S. Ciatto, V. Nelen, M. Kwiatkowski, A. Villers, A. Páez, S.M. Moss, M. Zappa, T.L.J. Tammela, T. Mäkinen, S. Carlsson, I.J. Korfage, M.-L. Essink-Bot, S.J. Otto, G. Draisma, C.H. Bangma, M.J. Roobol, F.H. Schröder, H.J. de Koning, Quality-of-Life Effects of Prostate-Specific Antigen Screening, New Engl. J. Med., 367 (2012) 595605.

[5] F.H. Schröder, J. Hugosson, M.J. Roobol, T.L.J. Tammela, S. Ciatto, V. Nelen, M. Kwiatkowski, M. Lujan, H. Lilja, M. Zappa, L.J. Denis, F. Recker, A. Páez, L. Määttänen, C.H. Bangma, G. Aus, S. Carlsson, A. Villers, X. Rebillard, T. van der Kwast, P.M. Kujala, B.G. Blijenberg, U.-H. Stenman, A. Huber, K. Taari, M. Hakama, S.M. Moss, H.J. de Koning, A. Auvinen, Prostate-Cancer Mortality at 11 Years of Follow-up, New Engl. J. Med., 366 (2012) 981-990.

[6] A.J. Vickers, C. Till, C.M. Tangen, H. Lilja, I.M. Thompson, An empirical evaluation of guidelines on prostate-specific antigen velocity in prostate cancer detection, J. Natl. Cancer Inst., 103 (2011) 462469.

[7] J. Wilbur, Prostate cancer screening: the continuing controversy, Am. Fam. Physician, 78 (2008) 13771384.

[8] S.P. Rowe, K.L. Gage, S.F. Faraj, K.J. Macura, T.C. Cornish, N. Gonzalez-Roibon, G. Guner, E. Munari, A.W. Partin, C.P. Pavlovich, M. Han, H.B. Carter, T.J. Bivalacqua, A. Blackford, D. Holt, R.F. Dannals, G.J. Netto, M.A. Lodge, R.C. Mease, M.G. Pomper, S.Y. Cho, F-18-DCFBC PET/CT for PSMA-Based Detection and Characterization of Primary Prostate Cancer, J. Nucl. Med., 56 (2015) 1003-1010.

[9] X. Tang, S.S. Mahajan, L.T. Nguyen, F. Beliveau, R. Leduc, J.A. Simon, V. Vasioukhin, Targeted inhibition of cell-surface serine protease Hepsin blocks prostate cancer bone metastasis, Oncotarget, 5 (2014) 1352-1362.

[10] K.A. Kelly, S.R. Setlur, R. Ross, R. Anbazhagan, P. Waterman, M.A. Rubin, R. Weissleder, Detection of early prostate cancer using a hepsin-targeted imaging agent, Cancer Res., 68 (2008) 2286-2291.

[11] S.R. Banerjee, M. Pullambhatla, C.A. Foss, A. Falk, Y. Byun, S. Nimmagadda, R.C. Mease, M.G. Pomper, Effect of Chelators on the Pharmacokinetics of Tc-99m-Labeled Imaging Agents for the 
Prostate-Specific Membrane Antigen (PSMA), J. Med. Chem., 56 (2013) 6108-6121.

[12] Y. Chen, M. Pullambhatla, C.A. Foss, Y. Byun, S. Nimmagadda, S. Senthamizhchelvan, G. Sgouros, R.C. Mease, M.G. Pomper, 2-(3-\{1-Carboxy-5-[(6-[F-18]Fluoro-Pyridine-3-Carbonyl)-Amino]Pentyl \}-Ureido)-Pentanedioic Acid, [F-18]DCFPyL, a PSMA-Based PET Imaging Agent for Prostate Cancer, Clin. Cancer. Res., 17 (2011) 7645-7653.

[13] S.R. Banerjee, M. Pullambhatla, Y. Byun, S. Nimmagadda, G. Green, J.J. Fox, A. Horti, R.C. Mease, M.G. Pomper, Ga-68-Labeled Inhibitors of Prostate-Specific Membrane Antigen (PSMA) for Imaging Prostate Cancer, J. Med. Chem., 53 (2010) 5333-5341.

[14] H.M. Shallal, I. Minn, S.R. Banerjee, A. Lisok, R.C. Mease, M.G. Pomper, Heterobivalent agents targeting PSMA and integrin-alphavbeta3, Bioconjug. Chem., 25 (2014) 393-405.

[15] A. Ghosh, W.D. Heston, Tumor target prostate specific membrane antigen (PSMA) and its regulation in prostate cancer, J. Cell. Biochem., 91 (2004) 528-539.

[16] S.R. Banerjee, C.A. Foss, M. Castanares, R.C. Mease, Y. Byun, J.J. Fox, J. Hilton, S.E. Lupold, A.P. Kozikowski, M.G. Pomper, Synthesis and Evaluation of Technetium-99m- and Rhenium-Labeled Inhibitors of the Prostate-Specific Membrane Antigen (PSMA), J. Med. Chem., 51 (2008) 4504-4517.

[17] M.I. Davis, M.J. Bennett, L.M. Thomas, P.J. Bjorkman, Crystal structure of prostate-specific membrane antigen, a tumor marker and peptidase, Proc.Natl. Acad. Sci. U. S. A., 102 (2005) 59815986.

[18] J.R. Somoza, J.D. Ho, C. Luong, M. Ghate, P.A. Sprengeler, K. Mortara, W.D. Shrader, D. Sperandio, H. Chan, M.E. McGrath, B.A. Katz, The Structure of the Extracellular Region of Human Hepsin Reveals a Serine Protease Domain and a Novel Scavenger Receptor Cysteine-Rich (SRCR) Domain, Structure, 11 (2003) 1123-1131.

[19] S.M. Dhanasekaran, T.R. Barrette, D. Ghosh, R. Shah, S. Varambally, K. Kurachi, K.J. Pienta, M.A. Rubin, A.M. Chinnaiyan, Delineation of prognostic biomarkers in prostate cancer, Nature, 412 (2001) 822-826.

[20] K.A. Kelly, S.R. Setlur, R. Ross, R. Anbazhagan, P. Waterman, M.A. Rubin, R. Weissleder, Detection of early prostate cancer using a hepsin-targeted imaging agent, Cancer Res., 68 (2008) 2286-2291.

[21] O. Klezovitch, J. Chevillet, J. Mirosevich, R.L. Roberts, R.J. Matusik, V. Vasioukhin, Hepsin promotes prostate cancer progression and metastasis, Cancer Cell, 6 (2004) 185-195.

[22] K.A. Landers, M.J. Burger, M.A. Tebay, D.M. Purdie, B. Scells, H. Samaratunga, M.F. Lavin, R.A. Gardiner, Use of multiple biomarkers for a molecular diagnosis of prostate cancer, International journal of cancer. Int. J. Cancer, 114 (2005) 950-956.

[23] V.N. Talesa, C. Antognelli, C. Del Buono, F. Stracci, M.R. Serva, E. Cottini, E. Mearini, Diagnostic potential in prostate cancer of a panel of urinary molecular tumor markers, Cancer Biomark., 5 (2009) 
241-251.

[24] M. Eder, M. Schafer, U. Bauder-Wust, U. Haberkorn, M. Eisenhut, K. Kopka, Preclinical evaluation of a bispecific low-molecular heterodimer targeting both PSMA and GRPR for improved PET imaging and therapy of prostate cancer, Prostate, 74 (2014) 659-668.

[25] B.A. Katz, C. Luong, J.D. Ho, J.R. Somoza, E. Gjerstad, J. Tang, S.R. Williams, E. Verner, R.L. Mackman, W.B. Young, P.A. Sprengeler, H. Chan, K. Mortara, J.W. Janc, M.E. McGrath, Dissecting and designing inhibitor selectivity determinants at the S1 site using an artificial Ala190 protease (Ala190 uPA), J. Mol. Biol., 344 (2004) 527-547.

[26] M. Subedi, J. Chen, E. Kang, K.I. Kim, Y. Byun, Facile Synthesis of 2-(4-Hydroxybiphenyl-3-yl)1H-indoles from Anilines and 5'-Bromo-2'-hydroxyacetophenone, Synth. Commun., 45 (2015) 17041709.

[27] J.H. Forsberg, V.T. Spaziano, T.M. Balasubramanian, G.K. Liu, S.A. Kinsley, C.A. Duckworth, J.J. Poteruca, P.S. Brown, J.L. Miller, Use of lanthanide(III) ions as catalysts for the reactions of amines with nitriles, J. Org. Chem., 52 (1987) 1017-1021.

[28] R.A. Moss, W. Ma, D.C. Merrer, S. Xue, Conversion of 'obstinate' nitriles to amidines by Garigipati's reaction, Tetrahedron Lett. 36 (1995) 8761-8764.

[29] S. Chandrappa, H. Chandru, A.C. Sharada, K. Vinaya, C.S. Ananda Kumar, N.R. Thimmegowda, P. Nagegowda, M. Karuna Kumar, K.S. Rangappa, Synthesis and in vivo anticancer and antiangiogenic effects of novel thioxothiazolidin-4-one derivatives against transplantable mouse tumor, Med. Chem. Res., 19 (2010) 236-249.

[30] M.L. Quan, P.C. Wong, C. Wang, F. Woerner, J.M. Smallheer, F.A. Barbera, J.M. Bozarth, R.L. Brown, M.R. Harpel, J.M. Luettgen, P.E. Morin, T. Peterson, V. Ramamurthy, A.R. Rendina, K.A. Rossi, C.A. Watson, A. Wei, G. Zhang, D. Seiffert, R.R. Wexler, Tetrahydroquinoline derivatives as potent and selective factor XIa inhibitors, J. Med. Chem., 57 (2014) 955-969.

[31] K.P. Maresca, S.M. Hillier, F.J. Femia, D. Keith, C. Barone, J.L. Joyal, C.N. Zimmerman, A.P. Kozikowski, J.A. Barrett, W.C. Eckelman, J.W. Babich, A Series of Halogenated Heterodimeric Inhibitors of Prostate Specific Membrane Antigen (PSMA) as Radiolabeled Probes for Targeting Prostate Cancer, J. Med. Chem., 52 (2009) 347-357.

[32] R.L. Mackman, B.A. Katz, J.G. Breitenbucher, H.C. Hui, E. Verner, C. Luong, L. Liu, P.A. Sprengeler, Exploiting subsite S1 of trypsin-like serine proteases for selectivity: Potent and selective inhibitors of urokinase-type plasminogen activator, J. Med. Chem., 44 (2001) 3856-3871.

[33] Y. Chen, S. Dhara, S.R. Banerjee, Y. Byun, M. Pullambhatla, R.C. Mease, M.G. Pomper, A low molecular weight PSMA-based fluorescent imaging agent for cancer, Biochem. Biophys. Res. Commun., 390 (2009) 624-629. 
Scheme 1. Synthesis of amidine-containing biphenylindole analogs<smiles>CC(=O)c1cc(Br)ccc1O</smiles>

1

2

3<smiles>N/C(=N\O)c1ccc2[nH]c(-c3cc(Br)ccc3O)cc2c1</smiles>

4

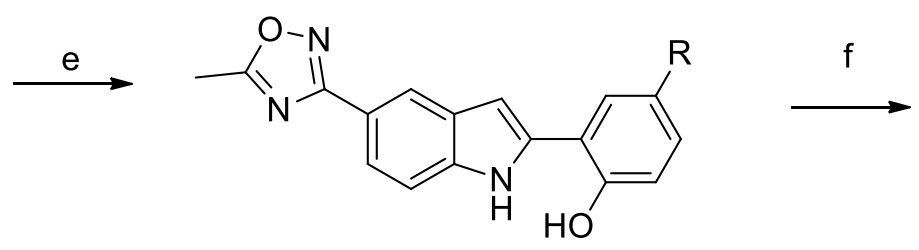

6: $\mathrm{R}=4-\mathrm{Cl}-\mathrm{Ph}$

7: $R=2$-thiophene

8: $\mathrm{R}=4-\mathrm{F}-\mathrm{Ph}$

9: $\mathrm{R}=4-\mathrm{CH}_{2} \mathrm{NHBOC}-\mathrm{Ph}$

10: $\mathrm{R}=\mathrm{Ph}$

11: $\mathrm{R}=4-\mathrm{OMe}-\mathrm{Ph}$

12: $\mathrm{R}=4-\mathrm{CF}_{3}-\mathrm{Ph}$<smiles>CC1=NC(c2ccc3[nH]c(-c4cc(Br)ccc4O)cc3c2)=CC1</smiles>

5<smiles>[R]c1ccc(O)c(-c2cc3cc(C(=N)N)ccc3[nH]2)c1</smiles>

13: $\mathrm{R}=4-\mathrm{Cl}-\mathrm{Ph}$

14: $R=2$-thiophene

15: $\mathrm{R}=\mathrm{H}$

16: $\mathrm{R}=4-\mathrm{F}-\mathrm{Ph}$

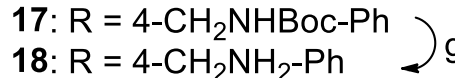

19: $\mathrm{R}=\mathrm{Ph}$

20: $\mathrm{R}=4-\mathrm{OMe}-\mathrm{Ph}$

21: $\mathrm{R}=4-\mathrm{CF}_{3}-\mathrm{Ph}$

Reagents and conditions: (a) 4-aminobenzonitrile, piperidine, toluene, $130{ }^{\circ} \mathrm{C}, 6 \mathrm{hr}$; (b) $\mathrm{Bu}_{4} \mathrm{NBr}$ (2 eq), $\mathrm{Pd}(\mathrm{OAc})_{2}(20 \mathrm{~mol} \%), \mathrm{DMSO}, 110{ }^{\circ} \mathrm{C}, 12 \mathrm{hr}$; (c) $\mathrm{NH}_{2} \mathrm{OH}-\mathrm{HCl}(10 \mathrm{eq}), \mathrm{Na}_{2} \mathrm{CO}_{3}$ (5 eq), EtOH, $90{ }^{\circ} \mathrm{C}, 7 \mathrm{hr}$; (d) NaOEt (2 eq), EtOH/ethylacetate (9/1), $90{ }^{\circ} \mathrm{C}, 2 \mathrm{hr}$; (e) appropriate boronic acid, $\mathrm{Cs}_{2} \mathrm{CO}_{3}, \mathrm{Pd}\left(\mathrm{PPh}_{3}\right)_{4}, \mathrm{DMF} / \mathrm{H}_{2} \mathrm{O}(5 / 1), 100{ }^{\circ} \mathrm{C}, 4 \mathrm{hr}$; (f) Raney-Ni/ $\mathrm{H}_{2}, \mathrm{MeOH} / \mathrm{AcOH}$ (7/1), $6 \mathrm{hr},(\mathrm{g}) 50 \%$ TFA in $\mathrm{CH}_{2} \mathrm{Cl}_{2}$, rt, $1 \mathrm{hr}$ 
Scheme 2. Synthesis of oxadiazole-protected PSMA-hepsin conjugate 27

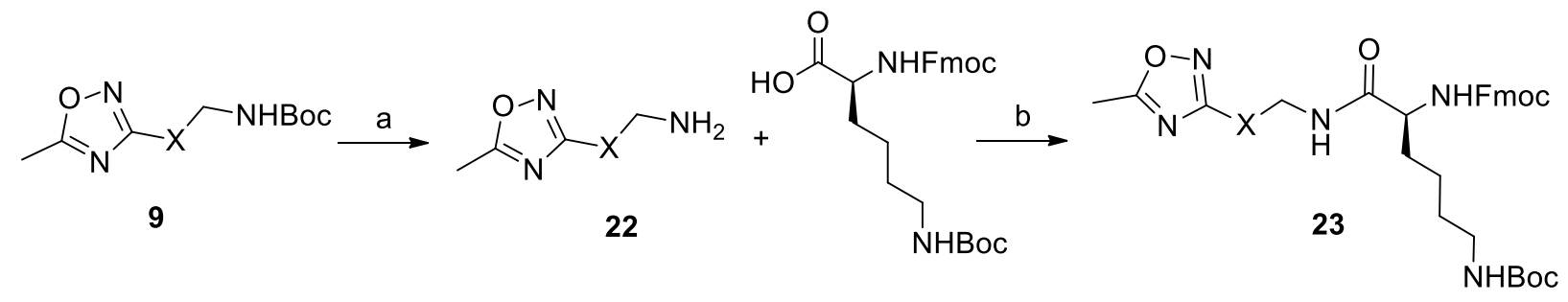

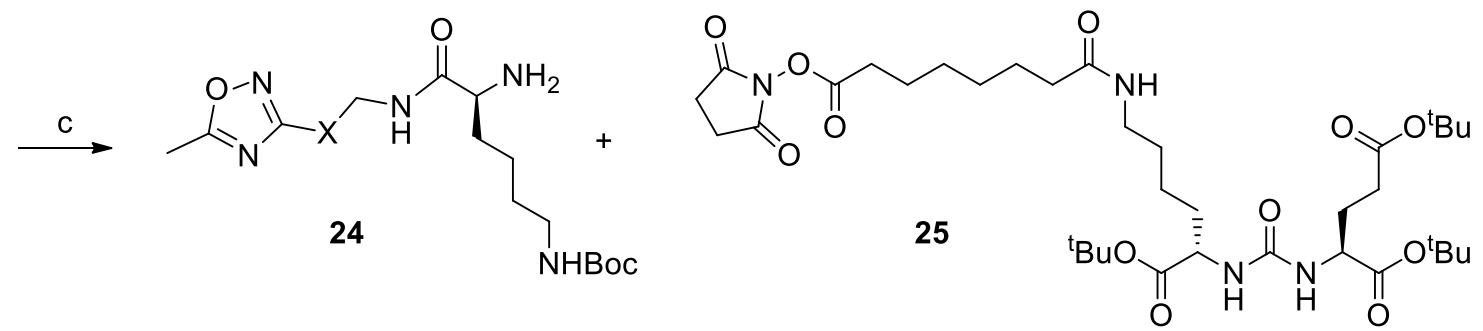<smiles>[AlH2][TeH]</smiles>

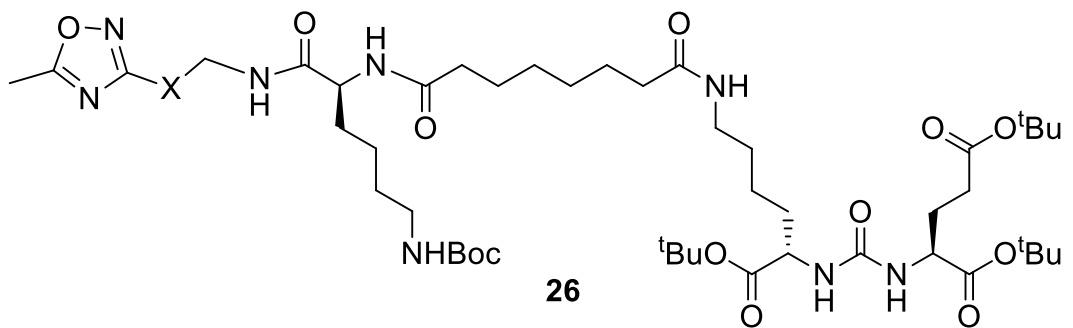

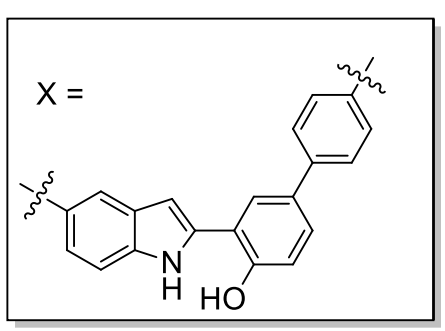<smiles>[X]CNC(=O)C(CCCCN)NC(=O)CCCCCCC(=O)NCCCC[C@H](NC(=O)NC(CCC(=O)O)C(=O)O)C(=O)O</smiles>

Reagents and conditions: (a) 50\% TFA in DCM, rt, 1 hr; (b) HATU (2 eq), $\mathrm{Et}_{3} \mathrm{~N}$ (2 eq), DMF, rt, $8 \mathrm{hr}$; (c) 25\% piperidine in DMF, rt, $1 \mathrm{hr}$; (d) Et $3 \mathrm{~N}$, DMF, rt, $1 \mathrm{hr}$; (e) $50 \%$ TFA in DCM, rt, $2 \mathrm{hr}$ 
Scheme 3. Synthesis of PSMA-hepsin conjugate $\mathbf{3 0}$ labeled with Sulfo-Cy7<smiles>[Z7]CCCCCC(=O)NC(CCCCN)C(=O)NCc1ccc(-c2ccc(O)c(-c3cc4cc([R])ccc4[nH]3)c2)cc1</smiles><smiles>[Y]C(C)(N)C(=N)N=C(C)C(C)(C)N=C(C)O</smiles>

Reagents and conditions: (a) Raney-Ni/ $\mathrm{H}_{2}(50 \mathrm{psi})$, dioxane/ $\mathrm{H}_{2} \mathrm{O} / \mathrm{AcOH}(6: 2: 1), 4 \mathrm{hr}$; (b) SulfoCy7-NHS ester, Tris-HCl $(0.1 \mathrm{M}, \mathrm{pH}=8.5)$, rt, $4 \mathrm{hr}$ 
Table 1. $\mathrm{IC}_{50}$ value of the prepared amidine-functionalized analogs and the crystal ligand CA-14

\begin{tabular}{cc}
\hline Compound & $\mathrm{IC}_{50}(\mu \mathrm{M})$ \\
\hline $\mathbf{1 3}$ & $23 \pm 2.4$ \\
$\mathbf{1 4}$ & $70 \pm 6.4$ \\
$\mathbf{1 5}$ & $44 \pm 3.8$ \\
$\mathbf{1 6}$ & $5.9 \pm 0.5$ \\
$\mathbf{1 7}$ & $11 \pm 1.5$ \\
$\mathbf{1 8}$ & $9.3 \pm 0.4$ \\
$\mathbf{1 9}$ & $8.9 \pm 2.8$ \\
$\mathbf{2 0}$ & $7.3 \pm 0.3$ \\
$\mathbf{2 1}$ & $14 \pm 0.8$ \\
$\mathbf{3 0}$ & $2.8 \pm 0.9$ \\
$\mathrm{CA}-14$ & $2.6 \pm 0.1$ \\
\hline
\end{tabular}

Figure Legends

Figure 1. (a) Binding of CA-14 (original crystal ligand) to hepsin (b) Expanded view showing hydrogen bonding of CA-14 to amino acid (c) Binding of 19 to hepsin (d) Expanded view showing hydrogen bonding of $\mathbf{1 9}$ to amino acid

Figure 2. $\mathrm{IC}_{50}$ curves of $\mathbf{3 0}$ and $\mathbf{3 1}$ to inhibit PSMA

Figure 3. In vitro uptake of $\mathbf{3 0}$ and $\mathbf{3 1}$ in PC3/ML, PC3/ML-PSMA, PC3/ML-HPN and PC3/ML-PSMA-HPN cell lines

Figure 4. In vivo near infrared imaging of $\mathbf{3 0}$ in mice harboring PC3/ML-PSMA (red circles) and PC3/ML-PSMA-HPN (white circles) tumors. Figures A and B represent two independent experiments. 
Figure 1
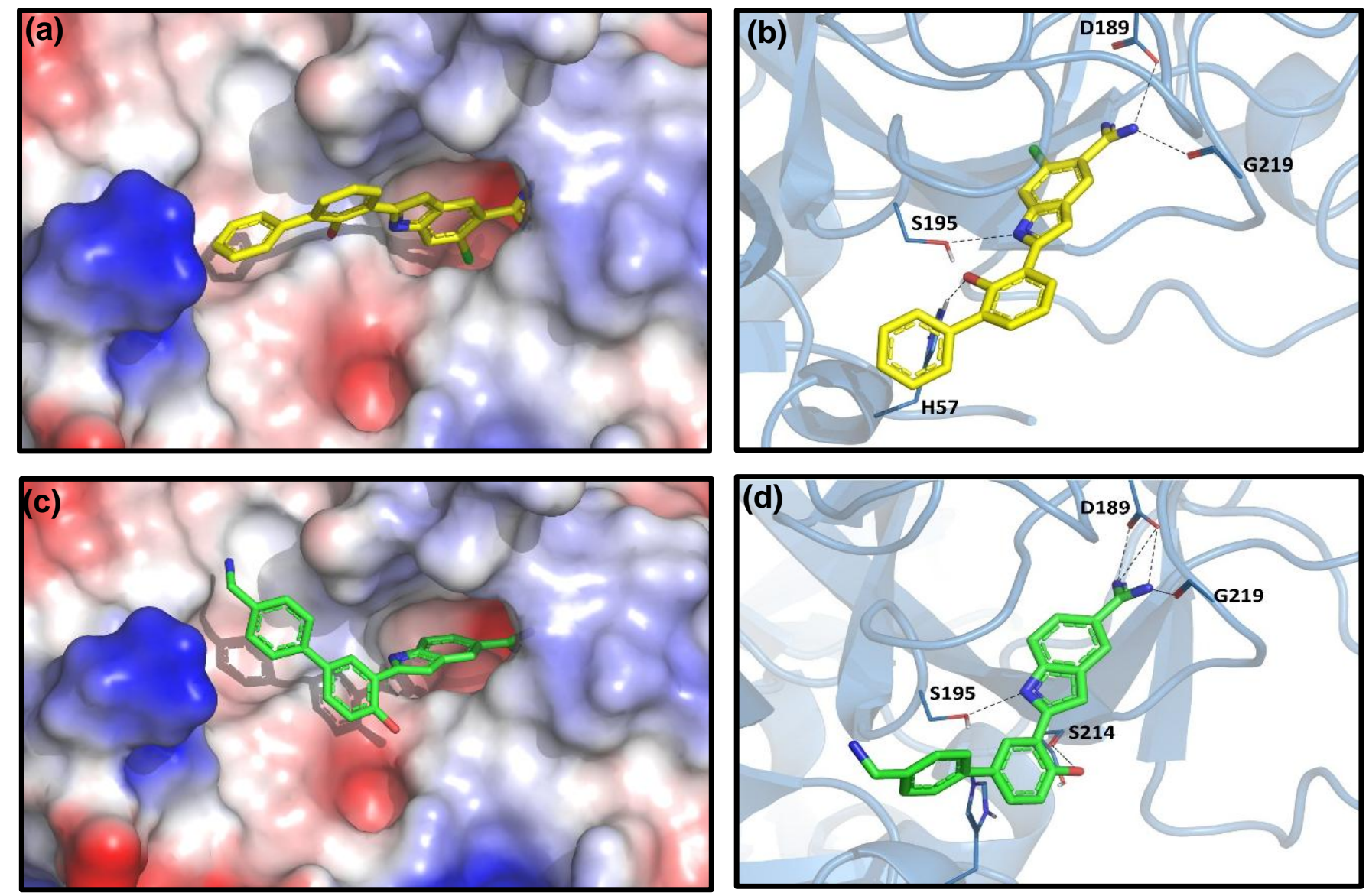
Figure 2

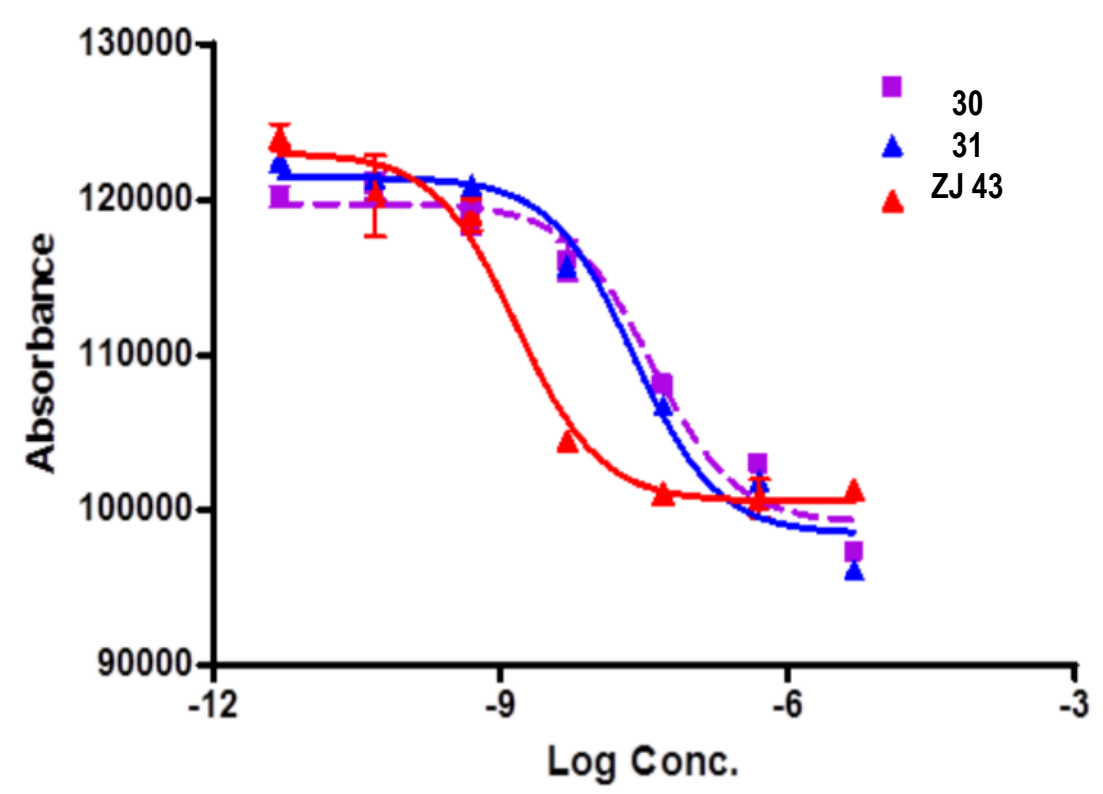




\section{Figure 3}
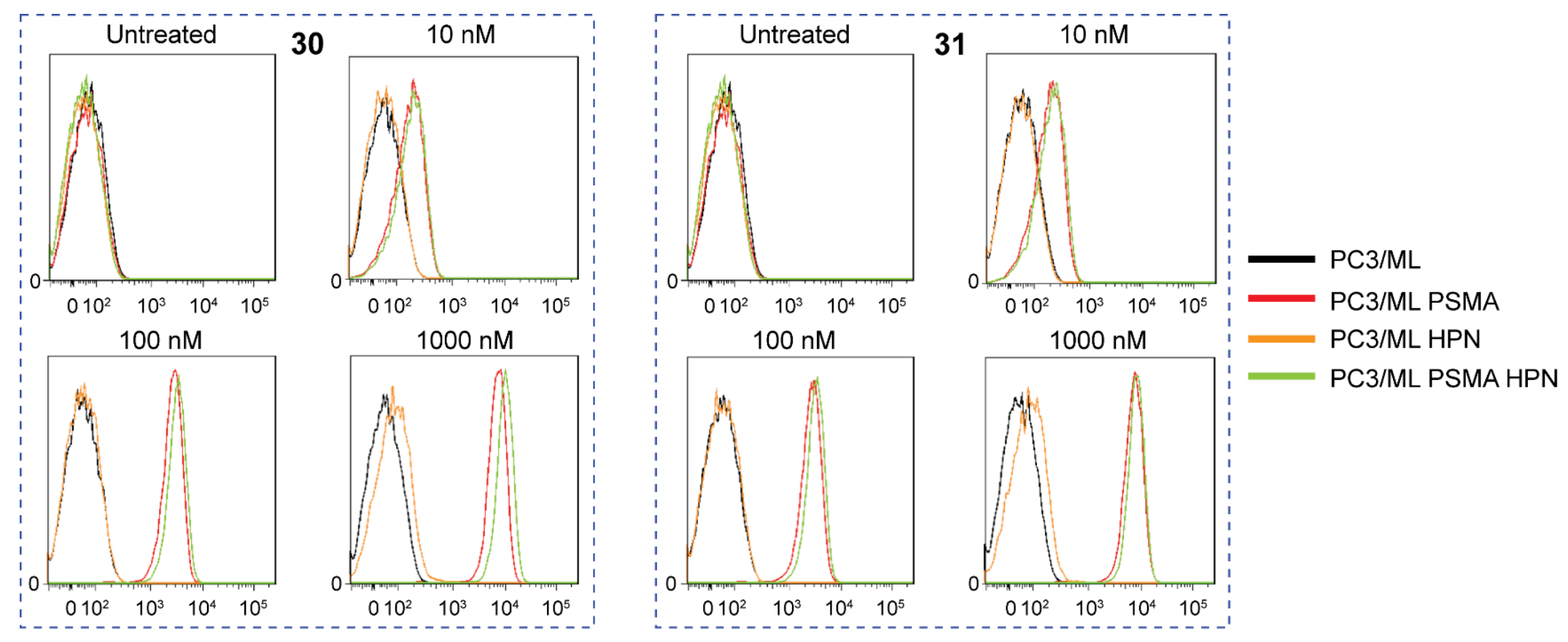
Figure 4

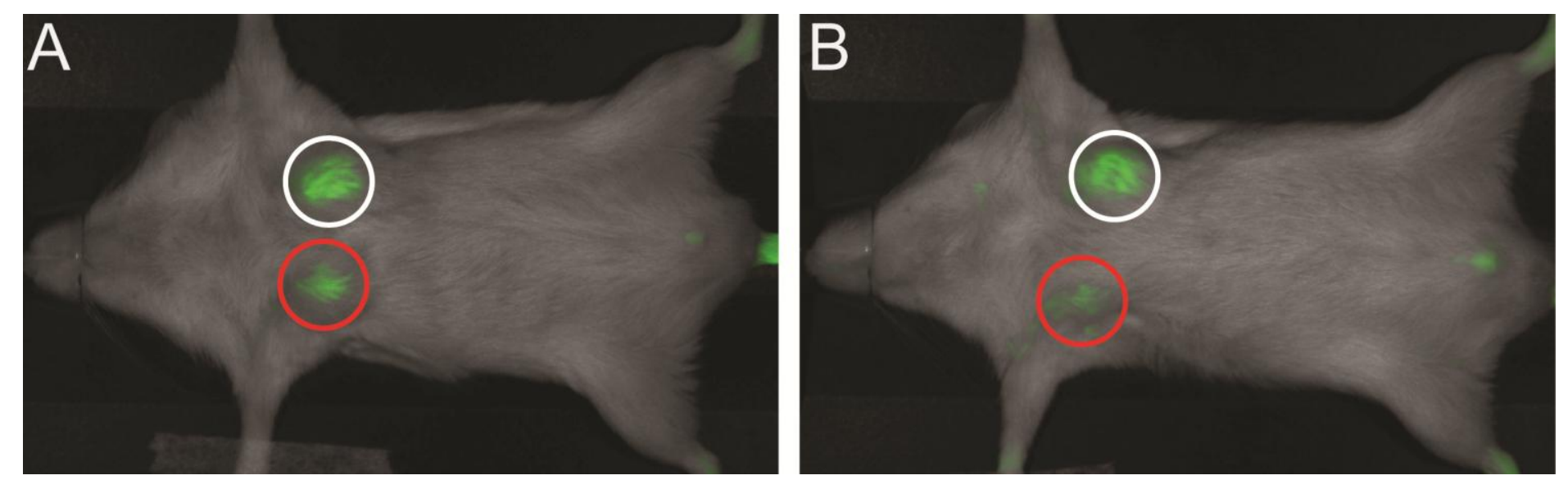



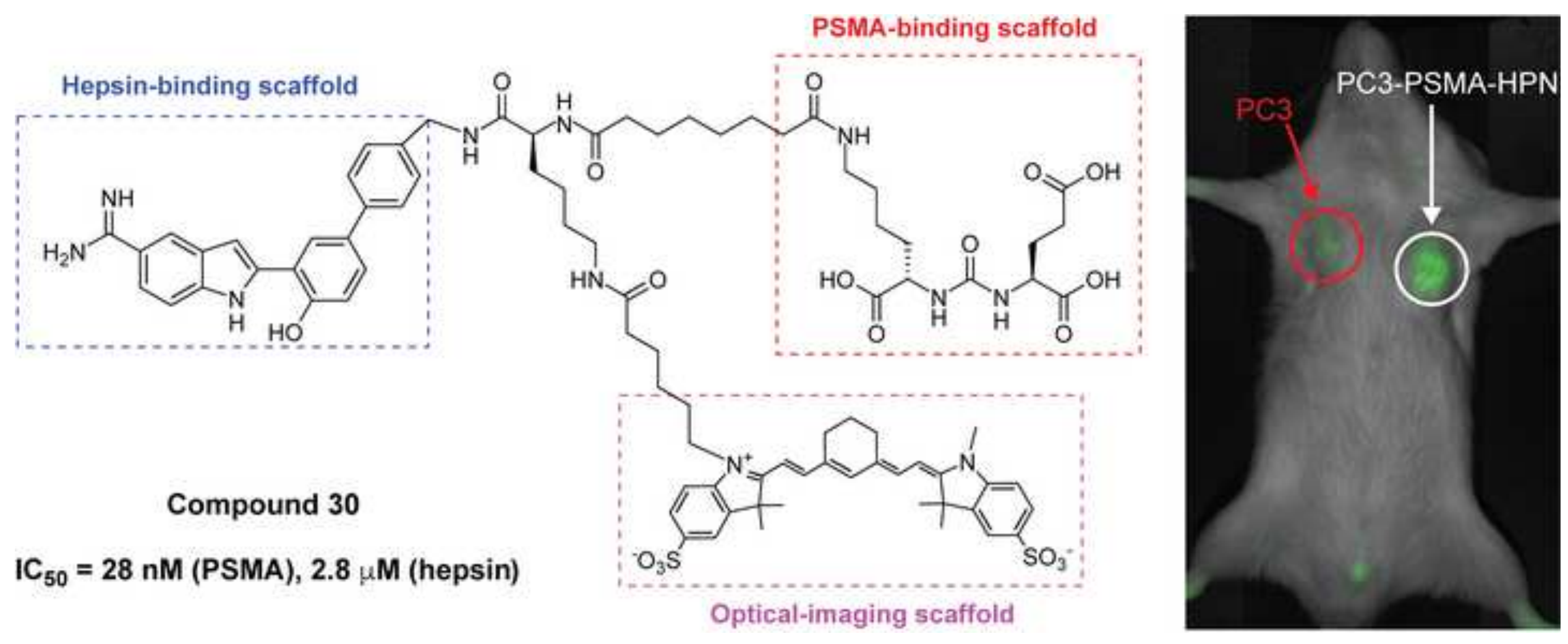